\title{
Planetary Nebulae Detected in the Spitzer Space Telescope GLIMPSE II Legacy Survey ${ }^{1}$
}

\author{
Yong Zhang \& Sun Kwok \\ Department of Physics, University of Hong Kong, Pokfulam Road, Hong Kong \\ zhangy96@hku.hk; sunkwok@hku.hk
}

\begin{abstract}
We report the result of a search for the infrared counterparts of 37 planetary nebulae (PNs) and PN candidates in the Spitzer Galactic Legacy Infrared Mid-Plane Survey Extraordinaire II (GLIMPSE II) survey. The photometry and images of these PNs at 3.6, 4.5, 5.8, 8.0, and $24 \mu \mathrm{m}$, taken through the Infrared Array Camera (IRAC) and the Multiband Imaging Photometer for Spitzer (MIPS), are presented. Most of these nebulae are very red and compact in the IRAC bands, and are found to be bright and extended in the $24 \mu \mathrm{m}$ band. The infrared morphology of these objects are compared with $\mathrm{H} \alpha$ images of the Macquarie-AAO-Strasbourg (MASH) and MASH II PNs. The implications for morphological difference in different wavelengths are discussed. The IRAC data allow us to differentiate between PNs and H II regions and be able to reject non-PNs from the optical catalogue (e.g., PNG 352.1-00.0). Spectral energy distributions (SEDs) are constructed by combing the IRAC and MIPS data with existing near-, mid-, and far-IR photometry measurements. The anomalous colors of some objects allow us to infer the presence of aromatic emission bands. These multi-wavelength data provide useful insights into the nature of different nebular components contributing to the infrared emission of PNs.
\end{abstract}

Subject headings: infrared: ISM — planetary nebulae: general — stars: AGB and post-AGB

\footnotetext{
${ }^{1}$ Figures $2-43$ are available on the ApJ website or on request from the authors.
} 


\section{INTRODUCTION}

The traditional view of planetary nebulae $(\mathrm{PNs})$ is that it is a shell of gas ionized by a hot central star and exhibits an emission-line spectrum. The theoretical understanding that PNs descend from asymptotic giant branch (AGB) stars and should contain remnants of the dust envelope led to the realization that PNs should also be bright infrared objects (Kwok 1982). This was confirmed after the Infrared Astronomical Satellite (IRAS) all-sky survey found that over 1000 PNs show far infrared emission (Pottasch et al. 1984; Zhang \& Kwok 1991). The typical color temperature of the dust component in PNs is between 100 and 200 $\mathrm{K}$, warmer than the dust component in $\mathrm{H}$ II regions but cooler than the circumstellar dust envelopes of AGB stars. This is consistent with the fact that the dust envelopes in PNs represent the dispersing remnants of the AGB envelopes (Kwok 1990). Infrared emission is now recognized as a defining observational properties of PNs.

However, the location of the infrared emitting dust component remains poorly known. Only recently has the angular resolution of mid-infrared cameras on large ground-based telescopes with adaptive optics become high enough to image the distribution of dust in PNs. Space observations therefore offer a better opportunity to survey a large number of PNs. The Infrared Array Camera (IRAC) on the Spitzer Space Telescope has an angular resolution $<2$ arcsec (Fazio et al. 2004) and its capability in imaging the dust component of PNs has been clearly demonstrated by Hora et al. (2004). Since the peak of the dust emission from PNs occurs at wavelengths $>20 \mu \mathrm{m}$, the Multiband Imaging Photometer for Spitzer (MIPS; Rieke et al. 2004), although offering less angular resolution than IRAC, would provide useful information on the distribution of the main cold dust component. Based on the MIPS observations at 24 and $70 \mu \mathrm{m}$, Su et al. (2007) found possible evidence for the presence of a debris disk around the central star of the Helix Nebula. Other examples of PNs studied by Spitzer includes NGC 2346 (Su et al. 2004), NGC 650 (Ueta 2006), and a sample of PNs in the Large Magellanic Cloud (LMC; Hora et al. 2008).

One of the advantages of IR observations is that they are hardly affected by dust reddening and are therefore ideal to study PNs heavily obscured by interstellar dust. From the IRAC observations, Cohen et al. (2005a) identified an extremely red PN G313.3+00.3 which is optically invisible. This raises the possibility that there are many obscured PNs in the Galaxy, for which Spitzer IR observations could explore. A high-spatial-resolution and high-sensitivity $\mathrm{H} \alpha$ survey of the southern Galactic plane has been undertaken using the Anglo-Australiam Observatory UK Schmidt Telescope (AAO/UKST) to search for very faint PNs (Parker et al. 1999). This has led to the discovery of over 1000 new PNs and $\mathrm{PN}$ candidates (which we refer to hereafter simply as PNs). The results of this survey was published as the Macquarie/AAO/Strasbourg $\mathrm{H} \alpha$ Planetary Galactic Catalogue (MASH) 
(Parker et al. 2006) and the MASH II supplement (Miszalski et al. 2008). These new PNs are typically more obscured than known Galactic PNs and warrant further investigation at IR wavelengths.

The Spitzer GLIMPSE (Galactic Legacy Infrared Mid-Plane Survey Extraordinaire; see Benjamin et al. 2003 and Churchwell et al. 2009) survey provides IRAC images with high resolution and sensitivity at wavelengths from 3.6 to $8.0 \mu \mathrm{m}$, allowing us to perform high-quality infrared imaging and photometry of PNs. The first-release GLIMPSE I data cover a Galactic region of $|l|=10^{\circ}-65^{\circ}$ and $|b| \leq 1^{\circ}$ (total area $\sim 220$ square degrees). Comprehensive GLIMPSE I-based studies of PNs have been presented by some authors (e.g. Cohen et al. 2007b; Phillips \& Ramos-Larios 2008a,b; Ramos-Larios \& Phillips 2008 ). Kwok et al. (2008, hereafter Paper I) imaged 30 PN\&2 detected in the GLIMPSE I survey and constructed IR spectral energy distributions (SEDs) which clearly show a distinction between the photospheric, nebular bound-free, and dust emission components.

In this paper, we extend the work of Paper I to the GLIMPSE II survey area that covers the Galactic bulge regions. We present the photometry and images of $37 \mathrm{PNs}$ detected in the GLIMPSE II survey. These results are compared with those of the GLIMPSE I PNs. To better explore nebular dust emission, we also take into account the $24 \mu \mathrm{m}$ data from the Spitzer Legacy program MIPS Inner Galactic Plane Survey (MIPSGAL). This paper is organized as follows: in Section 2 we briefly introduce the GLIMPSE II observations and the Spitzer data used in this study; in Section 3 we describe the data processing; in Section 4 we present the Mid-IR images and our measurement results of fluxes, sizes, and SEDs for individual PNs; the astrophysical implications of our results are discussed in Section 5; and Section 6 summarizes the main conclusions.

\section{OBSERVATION}

The data used in this paper were taken from the GLIMPSE II Version 2.0 Data Release3. The GLIMPSE II survey covers longitudes within $10^{\circ}$ of the center regions of the Galaxy $\left(|b| \leq 1^{\circ}\right.$ for $|l|=10^{\circ}-5^{\circ},|b| \leq 1.5^{\circ}$ for $|l|=5^{\circ}-2^{\circ}$, and $|b| \leq 2^{\circ}$ for $|l|=2^{\circ}-0^{\circ}$; total area $\sim 54$ square degrees) at 3.6, 4.5, 5.8, and $8.0 \mu \mathrm{m}$ with resolutions from $1.6^{\prime \prime}-1.8^{\prime \prime}$, but excludes an area of $\sim 3$ square degrees at the Galactic center observed by the PID 3677 GO program (which however has been included in the GLIMPSE II v2.0 mosaics). The survey

\footnotetext{
${ }^{2}$ Note that some objects in Paper I are actually compact H II regions, but are referred to as 'PNs' for convenience of denotation (their non-PN nature has been discussed in text).

${ }^{3}$ see http://www.astro.wisc.edu/glimpse/glimpsedata.html.
} 
regions are roughly shown in Figure 1. The observations were made with three 1.2 second exposures at each position during the periods between September 2005 and April 2006. The procedures of data reduction are similar to those described in Paper I. The GLIMPSE II data products include mosaic images, a highly reliable Point Source Catalog (GLMIIC), and a more complete Point Source Archive (GLMIIA). Further details of the GLIMPSE II data can be found in Churchwell et al. (2007) and Meade et al. (2008).

We also made use of the data from the MIPSGAL survey 4. The MIPSGAL observations were carried out at several epochs between 2005-2006 utilizing the MIPS instrument aboard Spitzer, aiming at providing a longer-wavelength complement to the GLIMPSE survey. An area of 278 square degrees in the inner Galactic plane, completely overlapping the GLIMPSE II observations, was imaged at the 24 and $70 \mu \mathrm{m}$ bands. In this study, we only used the publicly available $24 \mu \mathrm{m}$ images of the same region as GLIMPSE II. These images have a basic sensitivity of $\sim 110 \mu \mathrm{Jy}$ and a field of view (FOV) of $\sim 5.4^{\prime} \times 5.4^{\prime}$. The detector for MIPS24 is $128 \times 128$ pixel Si:As arrays with a bandwidth of $4.7 \mu \mathrm{m}$. The $24 \mu \mathrm{m}$ mosaics have a saturation limit of $1700 \mathrm{MJy} / \mathrm{sr}$ for extended source and a resolution of $6{ }^{\prime \prime}$. The data calibration was performed by both the Spitzer Science Center (SSC) and the MIPSGAL team. A good summary of the current status of the MIPS24 data sets can be found in Cohen (2009). The data reported in this paper were taken from the MIPSGAL v3.0 Data Delivery (Carey et al. 2008), which provides the $24 \mu \mathrm{m}$ mosaics $\left(1.1^{\circ} \times 1.1^{\circ}\right.$ in size) for all the survey regions.

\section{DATA PROCESSING}

We compared the GLIMPSE II data sets with the Edinburgh/AAO/Strasbourg Catalogue of Galactic Planetary Nebulae (version 1.0; Parker et al. 2001) and the MASH/MASH II catalogue (hereafter referred to as MASH PNs). These optical catalogues include the new PNs identified in the AAO/Strasbourg H $\alpha$ survey of the Milky Way and a small percentage of previous known Galactic PNs. We first selected a subsample of $71 \mathrm{MASH}$ PNs lying in the GLIMPSE II survey region, among which 10 were taken from the MASH II catalogue. We then undertook careful visual examination of the GLIMPSE II images in all the four IRAC bands using the DS9 software packages. If a PN is visible at at least one of the bands, we extracted a portion of the four-band images centered on the coordinates of this PN and measured its integrated fluxes.

\footnotetext{
${ }^{4}$ see http://mipsgal.ipac.caltech.edu/.

${ }^{5}$ DS9 is developed by Smithsonian Astrophysical Observatory.
} 
The total fluxes of these PNs were obtained using the same method as in Paper I. We first deduced the total on- and off-source fluxes using two apertures which have an identical size and were respectively centered on the PNs and in a position near the sources. Then the sum of all the fluxes of sources in the point-source archive within each aperture was obtained and subtracted from the on- and off-source fluxes respectively. The PN fluxes were finally determined from the on-source net fluxes subtracted off-source net fluxes. We have taken care to avoid over- and under-subtraction of the fluxes of point sources. For saturated sources, we only estimated lower limits of the fluxes. Since most of the GLIMPSE II PNs have well-defined boundaries, we also measured the major and minor diameters of each PN. For a few sources which have diffuse structures, the sizes of the bright parts were roughly estimated.

The same procedure is also applied to the MIPS24 data. There are several MASH PNs which have prominent $24 \mu \mathrm{m}$ emission but have no IRAC counterparts. We do not include these PNs in this analysis. Given the fact that the MIPS24 images have a resolution about three times lower than the IRAC ones, in some cases field stars cannot be resolved from the PNs in the MIPS24 images, and thus the flux measurements should be treated with some caution. The $24 \mu \mathrm{m}$ mosaics show some artificial structures around bright sources. We have attempted to minimize the effects of artifacts on the measurements of fluxes and sizes. Some PNs show faint extend halos which are likely to be real. We did not take into account these faint halos in the flux estimation since the fluxes of these halos are negligible compared with those of the main nebular regions.

\section{RESULTS}

We find that 37 MASH PNs (including five from the MASH II catalogue) have visible IRAC counterparts, among which nine are previously known Galactic PNs (Table1). The detection rate of the GLIMPSE II PNs is $52 \%$, about three times higher than that of the GLIMPSE I PNs (18\%; Paper I). This is partly ascribed to the fact that the interstellar

extinction toward the Galactic center is heavier and thus the optical observations only revealed those relatively bright PNs within the GLIMPSE II field. Most of the GLIMPSE II PNs appear to be compact and spherical, probably suggesting that they are generally more distant than GLIMPSE I PNs although we cannot completely rule out the possibility that some GLIMPSE II PNs are young and intrinsically compact. All the GLIMPSE II PNs are strong $24 \mu \mathrm{m}$ sources, some of which are even saturated.

Figure. 1 gives the approximate survey coverage of the GLIMPSE I/II projects overlaid with the positions of MASH PNs. A total of 12 GLIMPSE II PNs are located within the 
regions of $|b| \leq 1^{\circ}$. This corresponds to a surface density of $N_{\mathrm{PN}}=3 \mathrm{PNs}$ per $10 \mathrm{deg}^{2}$, a factor of six lower than that of GLIMPSE II PNs in the regions of $|b|>1^{\circ}$, where $N_{\mathrm{PN}}=18 \mathrm{PNS}$ per $10 \mathrm{deg}^{2}$. This is due to the strong IR background emission which hampers the detections of those PNs close to the Galactic plane, as one can clearly see in Meade et al. (2007) (their Figure. 1). This suggests that IRAC counterparts are more easily found outside the galactic plane.

In Figure. 1 we also plot the coverage of the GLIMPSE 3D survey (PI: Benjamin; PID= 30507), which images 112 square degrees in a series of regions with higher Galactic latitudes in all the four IRAC bands aiming at investigating the vertical stellar and interstellar structure of the inner Galaxy. We suppose that more PNs can be discovered in the GLIMPSE 3D field and it is interesting to compare the properties of these PNs located at higher Galactic latitudes with those of the GLIMPSE I/II PNs. Our work on the PNs detected in the GLIMPSE 3D survey is in progress and will be the subject of a forthcoming paper.

The IRAC images of the $37 \mathrm{PNs}$ are displayed in Figure 2 38, where we also overplot the contours of $\mathrm{H} \alpha$ emission for comparison. The $24 \mu \mathrm{m}$ images of these objects are displayed in Figure 39 43, In order to better show the features at different bands, we have adjusted the imaging depths as well as contrasts to create these color images. It is evident from these figures that most of these PNs appear to be redder and more extended than the surrounding field stars. The mid-IR (MIR) photometry results are presented in Table. 2, The first column lists the source names which are designed as "PNG $l b$ " following the IAU convention. Column 2 and 3 give the source right ascensions and declinations (J2000), which are mostly taken from the MASH catalogues. In a few rare cases the MASH catalogues give incorrect coordinates (e.g. PNG $000.9+01.8$ ) the corrected values are given in this table. Column 4-8 give the spatially integrated fluxes in the $3.6-24 \mu \mathrm{m}$ bands. Due to the very bright background emission that tends to overwhelm the near-IR emission from the PNs, most of the PNs near the Galactic center were detected by IRAC only in the $8.0 \mu \mathrm{m}$ band. The measured source sizes are presented in Column 9-13. For comparison, column 14 gives the $\mathrm{H} \alpha$ sizes taken from the MASH catalogues. For the PNs that are detected in all the four IRAC bands, we calculated the 3.6-8.0 $\mu \mathrm{m}$ SED slopes using $\alpha_{\mathrm{IRAC}}=d \log \left(\lambda F_{\lambda}\right) / d \log (\lambda)$. The resultant $\alpha_{\text {IRAC }}$ values are given in column 15 .

\subsection{Spectral Energy Distributions}

To better constrain the IR spectral properties of the GLIMPSE II PNs, we have searched other data archives, including the Two Micron All Sky Survey (2MASS), Deep Near Infrared Survey of the Southern Sky (DENIS), Midcourse Space Experiment (MSX), and the IRAS 
Point Source Catalogue (PSC). Data from these catalogues together cover the spectral region from 0.82 to $100 \mu \mathrm{m}$. All the available images have been checked visually to ensure that the correct counterpart is used. Table 3 tabulates the magnitudes and fluxes of these measurements. As these other surveys all have lower sensitivities than IRAC, in general only the brightest sources in the GLIMPSE II sample are detected in the other surveys. Since IRAC also offers better angular resolution, all the sources are unresolved in these archives, with the only exception of PNG 352.1 - 00.0. As PNG 352.1 - 00.0 has a large angular size, the data given in these point source catalogues only represent the lower limits of fluxes. We therefore do not include this object in Table 3. Combining our IRAC and MIPS photometry with these archive data, we have constructed SEDs for the PNs listed in Table 3 and the results are displayed in Figure 44,

A glance at Figure 44 shows that almost all the GLIMPSEII PNs are red objects with a rising spectrum between 1 and $20 \mu \mathrm{m}$. Their SEDs are in general consistent with the presence of a cold dust component of temperature lower than $300 \mathrm{~K}$. This confirms the IRAS observations that PNs are generally surrounded with cold dust shells (Zhang \& Kwok 1991). Some sources have a separate emission component at short wavelengths $(<3 \mu \mathrm{m})$, which can be attributed to contributions from the photospheric, nebular bound-free, or hot dust emission components (see, Zhang \& Kwok 1991, for a detailed discussion of SEDs of PNs).

The SED slope $\alpha_{\text {IRAC }}$ can provide quantitative description of the SED shape. We have determined $\alpha_{\text {IRAC }}$ through least-squares fit to the four IRAC bands, and their values are listed in Table 2. The SED slopes for all the GLIMPSE II PNs are $\alpha_{\text {IRAC }}>-1$, substantially larger than those found for stellar photospheres (Lada et al. 2006; Chavarría et al. 2008). Based on their study of young stellar objects (YSOs), Chavarría et al. (2008) introduced a classification scheme: Class I for $\alpha_{\text {IRAC }}>0$ (star plus infalling envelope), Class II for $-2 \geq \alpha_{\mathrm{IRAC}} \leq 0$ (star plus a disk), and Class III for $\alpha_{\mathrm{IRAC}}<-2$ (post-T Tauri stars or main-sequence photospheres). According to this classification, we found that among the 20 PNs with $\alpha_{\text {IRAC }}$ measurements 80 percent are categorized as Class I (star with envelope), and 20 percent as Class II (star with disk). de la Fuente et al. (2008) determined the $\alpha_{\text {IRAC values }}$ for 19 ultracompact (UC) H II regions, which are comparable with those for PNs. Although further statistical work based on a larger PN sample needs to be performed to determine whether the classification of YSO can apply to PNs, we show here that $\alpha_{\text {IRAC }}$ can serve as a useful indicator to investigate nebular properties. Nevertheless, it should be borne in mind that the slopes of PNs' SEDs can be significantly affected by various emission features (as discussed below) and it is difficult to reach solid conclusions without spectroscopic studies.

Although the SEDs are useful to distinguish contributions from the photospheric, nebular, and dust components, the presence of various emission features complicates the IRAC/MIPS 
based studies of nebular IR properties. These possible emission features include: the contributions by the 3.3, 6.2 and $7.7 \mu \mathrm{m}$ aromatic infrared bands (AIB) to the 3.6, 5.8, and $8.0 \mu \mathrm{m}$ IRAC bands, molecular hydrogen vibrational-rotational bands to all the IRAC bands, recombination lines $(\operatorname{Pf} \gamma 3.7 \mu \mathrm{m}, \operatorname{Br} \alpha 4.05 \mu \mathrm{m}$, and $\operatorname{Pf} \alpha 7.5 \mu \mathrm{m})$ to the $3.6,4.5$, and $8.0 \mu \mathrm{m}$ bands, and atomic collisionally excited lines to the $4.5-24 \mu \mathrm{m}$ bands (see also Paper I). It is impossible to accurately estimate the contributions of these features without spectroscopy. The ISO spectrum of PNG 359.3-00.9 (Hb 5) is available (Pottasch \& Surendiranath 2007) and is plotted in Figure. 44, Figure. 45 gives an expanded view of this spectrum overlaid with the spectral response curves for the IRAC and MIPS $24 \mu \mathrm{m}$ bands. This figure shows that the contributions from these features to these bands are significantly large to change the MIR colors.

\subsection{MIR Colors}

In Figure 46 we plot the [3.6] - [4.5] versus [5.8] - [8.0] color-color diagram for the GLIMPSE II PNs. For comparison, this figure also plots the colors of the GLIMPSE I PNs reported in the literature (Paper I; Hora et al. 2004; Cohen et al. 2007a, b; Phillips \& Ramos-Larios 2008a, b). There is no obvious color difference between the GLIMPSE I and the GLIMPSE II PNs in this diagram. These PNs are very red and clustered around a position near $(2,1)$. For the PNs with MIR emission dominated by contributions from the central stars, the IRAC colors are close to the $(0,0)$ point. We have not attempted to correct for interstellar extinction towards these PNs (which is supposed to be small at IRAC wavelengths) but show a reddening vector of $A_{V}=10 \mathrm{mag}$ in this figure. We note that the [5.8] - [8.0] color index decreases with increasing reddening because extinction in the $8.0 \mu \mathrm{m}$ band is primarily caused by a silicate absorption feature peaking at $9.7 \mu \mathrm{m}$. Although one expects Galactic bulge PNs to suffer a higher degree of interstellar extinction, but this is not evident in Figure 46. The GLIMPSE II PNs are indistinguishable from the GLIMPSE I PNs in the color-color diagram. Furthermore, the data scattering direction in Figure 46 is roughly perpendicular to the reddening vector. We thus conclude that MIR extinction is negligible and does not affect the colors of the PNs in our sample. The IRAC colors of PNs mainly reflect the color temperatures and the relative contributions from different components including the photosphere, the ionized nebula, the dust envelop, and various emission features.

We have calculated the simulated colors using blackbody models convolved with the spectral response functions, and the results are plotted in Figure 46. Using the simulated blackbody colors as a reference, we can see that the [3.6] - [4.5] colors of PNs suggest significantly higher temperatures than those by the [5.8] - [8.0] colors. This is indicative of 
the substantial contribution of emission from AIB emissions, ionized gas, and/or photosphere to the $3.6 \mu \mathrm{m}$ band. The relative contribution of the latter two have been illustrated in Zhang \& Kwok (1991).

In spite of the fact that PNs have different colors with stellar objects, the color-color diagram alone does not enable to completely distinguish PNs from other MIR sources. From Figure 46 we can find that the colors for PNs partly overlap with those for Class I YSOs (i.e. star with envelope) and the PNs with strong emission from central stars might be confused with Class II YSOs (i.e. star with disk). Moreover, some H II regions are located within the same position as PNs in the color diagram. Therefore, despite the color-color diagram can serve as a tool to sample PN candidates, detailed investigations of morphologies and spectra are essentially required for accurate PN designation.

Figure 47 shows the [3.6] - [8.0] versus [8.0] - [24] color-color diagram. The [3.6] - [8.0] colors are in the range from $1-5$, and the [8.0] - [24] colors are in the range from 3-7. The IRAC-MIPS colors for these Galactic PNs are similar to those found for LMC PNs by Hora et al. (2008, see their Figure 5). For the same reason as in Figure 46, the PN colors cannot be explained by single-temperature blackbodies. Comparing Figure 47 with the colorcolor diagram for the members of the IC 348 cluster presented by Lada et al. (2006, see their Figure. 7), we find that the distribution of PNs in this diagram are clearly centered at a position redder than IC 348 cluster sources, even for the YSOs with thick disk. This suggests that the $24 \mu \mathrm{m}$ excess is a prominent characteristic of PNs.

In Fig. 48 we plot the IRAC color-magnitude diagrams ([3.6] versus [3.6] - [8.0] and [8.0] versus $[3.6]-[8.0])$. These diagrams show that the GLIMPSE II PNs generally have lower integrated fluxes than the GLIMPSE I PNs, although their colors are similar to each other. These diagrams can be compared with those for LMC PNs (Hora et al. 2008, Fiugre 6). We find that the distribution of Galactic PNs in the color-magnitude diagrams perfectly follows those of LMC PNs in shape although the galactic PNs are nearer and hence brighter.

\subsection{Individual Objects}

PNG 000.0-01.3. - This source was detected by IRAC in the 5.8 and $8.0 \mu \mathrm{m}$ bands only (Figure 21). Parker et al. (2006) classified it as a 'Likely' PN. The object was also detected by the NRAO VLA Sky Survey (NVSS; Condon et al. 1998), which measured a flux density of $4.5 \pm 0.8 \mathrm{mJy}$ at $1.4 \mathrm{GHz}$. In spite of its faintness, its $\mathrm{H} \alpha$ and $8.0 \mu \mathrm{m}$ images are visually similar and both reveal an oval structure.

PNG 000.1-01.7. - This source was detected by IRAC in the $8.0 \mu \mathrm{m}$ band only (Fig- 
ure 3). Its PN status has been confirmed by spectroscopy (designated as JaSt85; Jacoby \& Van de Steene 2004). Van de Steene \& Jacoby (2001) observed this Galactic bulge PN in the radio continuum at 3 and $6 \mathrm{~cm}$ and estimated a distance of $\sim 7.2 \mathrm{kpc}$. The NVSS survey gives a flux density of $2.9 \pm 0.6 \mathrm{mJy}$ at $1.4 \mathrm{GHz}$ (Condon et al. 1998). Given large [N II]/H $\alpha$ flux ratio (Parker et al. 2006), it might be a low excitation PN. It is extremely faint in the IRAC bands but fairly bright in the MIPS24 band. Albeit with an ambiguous boundary, the size of $8.0 \mu \mathrm{m}$ image is roughly consistent with that of $\mathrm{H} \alpha$.

PNG 000.3-01.6. - This source was detected by IRAC in the $8.0 \mu \mathrm{m}$ band only (Figure 4). Its PN status has been confirmed by spectroscopy (designated as JaSt86; Jacoby \& Van de Steene 2004). Based the radio observation, Van de Steene \& Jacoby (2001) estimated a distance of $6.6 \mathrm{kpc}$. The NVSS survey gives a flux density of $4.1 \pm 0.5 \mathrm{mJy}$ at $1.4 \mathrm{GHz}$ (Condon et al. 1998). It is very compact in all the MIR and $\mathrm{H} \alpha$ bands.

PNG 000.5+01.9. - This source was detected by IRAC in the $8.0 \mu \mathrm{m}$ band only (Figure 5). The object was first discovered by Kohoutek (1994) (designated as K 6-7), and its PN status was subsequently confirmed by spectroscopy (designated as JaSt17; Jacoby \& Van de Steene 2004). Van de Steene \& Jacoby (2001) estimated the distance to be $7.1 \mathrm{kpc}$. The NVSS survey gives a flux density of $6.8 \pm 0.9 \mathrm{mJy}$ at $1.4 \mathrm{GHz}$ (Condon et al. 1998). The $8.0 \mu \mathrm{m}$ and $\mathrm{H} \alpha$ images are visually similar and show a compact and oval structure.

PNG 000.9-01.0. - This source was detected by IRAC in the $8.0 \mu \mathrm{m}$ band only (Figure 6). It is classified as a 'True' PN in the MASH II catalogue. The MIR ( 8.0 and $24 \mu \mathrm{m})$ and $\mathrm{H} \alpha$ bands show a compact and round structure without a sharp boundary.

PNG 000.9+01.8. - This source was detected by IRAC in the $8.0 \mu \mathrm{m}$ band only (Figure 7). Parker et al. (2006) classified it as a 'True' PN. The optical image shows an oval structure. From the IRAC image, we note that the object seems to be a core surrounded by an ambiguous diffuse halo.

PNG 001.0-01.9. - This source was detected by IRAC in the $8.0 \mu \mathrm{m}$ band only (Figure 8). The object was first discovered by Kohoutek (2002) (designated as K 6-35) and classified as a 'True' PN by Parker et al. (2006). The NVSS survey gives a flux density of $4.8 \pm 0.6 \mathrm{mJy}$ at $1.4 \mathrm{GHz}$ (Condon et al. 1998). The H $\alpha$ image shows that it is a bright, compact, and slightly oval $\mathrm{PN}$ with faint outer halo. Its $8 \mu \mathrm{m}$ counterpart is nearly overwhelmed by bright background emission.

PNG 001.0+01.9. - This source was only marginally detected by IRAC in the $8.0 \mu \mathrm{m}$ band and was completely invisible in the other IRAC bands (Figure 91). Exter et al. (2004) determined chemical abundances of this object with optical spectroscopy and classified it as a Type I PN. The NVSS survey gives a flux density of $20.6 \pm 1.3 \mathrm{mJy}$ at $1.4 \mathrm{GHz}$ (Condon et al. 
1998). Zhang (1995) deduced the statistical distance to be $3.46 \mathrm{kpc}$. In their catalogue of narrow band images of PNs, Schwarz et al. (1992) presented H $\alpha$ and [O III] images of this $\mathrm{PN}$, which exhibit an oval appearance with a size of $74^{\prime \prime}$ and of $43^{\prime \prime}$, respectively. The $8.0 \mu \mathrm{m}$ image is too faint to obtain a reliable flux. This PN is different from others in this sample of PNs in that its emission is significantly more compact at $24 \mu \mathrm{m}$ than at $\mathrm{H} \alpha$.

PNG 001.6-01.1. - This source was detected by IRAC in the $8.0 \mu \mathrm{m}$ band only (Figure 10). Its PN status has been confirmed by spectroscopy (designated as JaSt97; Jacoby \& Van de Steene 2004). Van de Steene \& Jacoby (2001) estimated the distance to be $5.9 \mathrm{kpc}$. The $8.0 \mu \mathrm{m}$ and $\mathrm{H} \alpha$ images have a similar size and both exhibit a compact oval appearance.

PNG 002.0+00.7. - This source was detected by IRAC in all the four bands (Figure 11). The NVSS survey gives a flux density of $4.1 \pm 0.5 \mathrm{mJy}$ at $1.4 \mathrm{GHz}$ (Condon et al. 1998). It is classified as a 'True' PN in the MASH II catalogue. The PN appears to be a point source in all the bands. Its IRAC color is only slightly redder than stars. The SED indicates the presence of cold dust with $T<300 \mathrm{~K}$ (Figure 44).

PNG 002.1-00.9. - This source was detected by IRAC in all the four bands (Figure 12). Parker et al. (2006) classified it as a 'Likely' PN. It was also independently found by Kohoutek (2002). This PN is very bright in all the bands, and exhibits a compact and round appearance. The SED indicates a cold dust component with $T<300 \mathrm{~K}$ (Figure 44).

PNG 002.1-01.1. - This source was detected by IRAC in the 5.8 and $8.0 \mu \mathrm{m}$ bands only (Figure 13). It is classified as a 'Ture' PN in the MASH II catalogue. The NVSS survey gives a flux density of $2.1 \pm 0.5 \mathrm{mJy}$ at $1.4 \mathrm{GHz}$ (Condon et al. 1998). Its $\mathrm{H} \alpha$ image shows a seemingly bipolar structure, which however is hard to tell from its fuzzy $8 \mu \mathrm{m}$ image.

PNG 002.2+00.5. - This source was detected by IRAC in all the four bands (Figure 14). The NVSS survey gives a flux density of $15.2 \pm 0.7 \mathrm{mJy}$ at $1.4 \mathrm{GHz}$ (Condon et al. 1998). The $\mathrm{H} \alpha$ image exhibits an elliptical appearance with resolved internal structure. The IRAC data show that it is a very red PN and has a morphology visibly similar to that of the archetypal PN NGC 7027, i.e., a limb-brightened, bi-conical shell with enhanced emission at the equator (see Cox et al. 2002, for the details of the image of NGC 7027).

PNG 002.2-01.2. - This source was detected by IRAC in the 4.5, 5.8, and $8.0 \mu \mathrm{m}$ bands only (Figure 15). It is classified as a 'Ture' PN by Parker et al. (2006). Strong [N II] and weak [O III] lines were detected by Parker et al. (2006), suggesting that it is a low excitation PN. The NVSS survey gives a flux density of $2.1 \pm 0.5 \mathrm{mJy}$ at $1.4 \mathrm{GHz}$ (Condon et al. 1998). The $8.0 \mu \mathrm{m}$ and $\mathrm{H} \alpha$ appearances are similar to each other and show a compact and rectangular shaped structure. 
PNG 003.4+01.4.- This source is almost invisible in the IRAC bands (Figure 16). We only find an extremely faint counterpart in the $8.0 \mu \mathrm{m}$ band. Its $24 \mu \mathrm{m}$ counterpart appears to be a point source and is fainter compared to other GLIMPSE II PNs. The object is described by Parker et al. (2006) as having faint, semi-circular arc around faint central star, and is classified as a 'likely' PN. Albeit with faintness in optical and MIR bands, it has a sufficiently strong radio flux. The NVSS survey gives a flux density of $15.2 \pm 0.7 \mathrm{mJy}$ at $1.4 \mathrm{GHz}$ (Condon et al. 1998). Its faint $8.0 \mu \mathrm{m}$ image does not allow us to say much about its infrared structure.

PNG 003.5-01.2.- This source was detected by IRAC in all the four bands (Figure 17). Parker et al. (2006) detected strong [N II] and weak [O III] lines in the object and classified it as a 'Possible' PN, possibly a symbiotic star containing a Mira variable. The object has an IRAS counterpart, IRAS $17554-2628$. The NVSS survey gives a flux density of $13.7 \pm 0.6 \mathrm{mJy}$ at $1.4 \mathrm{GHz}$ (Condon et al. 1998). The IRAC and $24 \mu \mathrm{m}$ images exhibit a very red and bright point source. The SED implies the presence of dust with a temperature of $\sim 300 \mathrm{~K}$ (Figure 44). Moreover, the increase of flux at $\sim 100 \mu \mathrm{m}$ points to the existence of another dust component with even lower temperature.

PNG 003.5+01.3. - This source was detected by IRAC in all the four bands (Figure 18). It is classified as a 'True' PN in the MASH II catalogue. The NVSS survey gives a flux density of $24.9 \pm 0.9 \mathrm{mJy}$ at $1.4 \mathrm{GHz}$ (Condon et al. 1998). Its IRAC images appear to be a point source. The SED shows a marked increase of flux from 6-12 $\mu \mathrm{m}$, suggesting the presence of cold dust of $T<300 \mathrm{~K}$ (Figure 44).

PNG 003.6-01.3.- This source was detected by IRAC in all the four bands (Figure 19). It is described by Parker et al. (2006) as a small, bright, round, and 'True' PN with strong [O III] and $\mathrm{H} \alpha$ emission. The IRAC images exhibit a similar appearance with $\mathrm{H} \alpha$ image although the former seem to be more compact. The SED shows an emission peak around $1.5 \mu \mathrm{m}$ (Figure 44), probably pointing to the substantial contribution from photospheric and nebular bound-free emission.

PNG 003.7+00.5.- This source is badly blended with a very bright star (Figure 20) which saturates IRAC. Nevertheless, we can still find evidence showing extended emission in the northwest of this bright field star. The object is described by Parker et al. (2006) as a very small, isolated slightly oval nebula with asymmetric enhance opposing edges, and is classified as a 'Likely' PN. The $24 \mu \mathrm{m}$ emission is seriously polluted by the bright field star, and thus we only give the upper limit of the flux.

PNG 004.3-01.4.- This source was detected by IRAC in all the four bands (Figure21). It is classified as a 'True' PN by Parker et al. (2006). The NVSS survey gives a flux density of 
$12.7 \pm 0.7 \mathrm{mJy}$ at $1.4 \mathrm{GHz}$ (Condon et al. 1998). This object has a IRAS counterpart, IRAS $17582-2553$. The MIPS $24 \mu \mathrm{m}$ flux of $1.7 \mathrm{Jy}$ is in excellent agreement with the $25 \mu \mathrm{m}$ flux (1.9 Jy) given in the IRAS PSC. Kistiakowsky \& Helfand (1995) imaged this PN through narrowband filter centered at [S III] $\lambda 9532$, which reveals a size of $\sim 2^{\prime \prime}$. The IRAC and $\mathrm{H} \alpha$ images display a compact and roughly round structure. The SED shows a steep increase of flux at $10 \mu \mathrm{m}$ (Figure 44), and suggests that the PN might have a dust component with a color temperature of $\sim 300 \mathrm{~K}$.

PNG 004.8-01.1. - This source was detected by IRAC in all the four bands (Figure 22). It is classified as a 'True' PN by Parker et al. (2006). This object has a IRAS counterpart, IRAS 17581 - 2522. The [S III] $\lambda 9532$ narrowband image suggests a size of $4^{\prime \prime}$ (Kistiakowsky \& Helfand 1995). The smaller size at [S III] compared to those at MIR and $\mathrm{H} \alpha$ bands is due to the fact that the former only traces the inner high-ionization regions. The NVSS survey gives a flux density of $16.2 \pm 3.1 \mathrm{mJy}$ at $1.4 \mathrm{GHz}$ (Condon et al. 1998). The $8 \mu \mathrm{m}$ and $\mathrm{H} \alpha$ images show an oval appearance. The SED suggests the presence of cold dust with $T<150 \mathrm{~K}$ (Figure 44).

PNG 006.1+00.8. - This source was detected by IRAC in all the four bands (Figure 23). It is classified as a 'True' PN by Parker et al. (2006). The $8 \mu \mathrm{m}$ and $\mathrm{H} \alpha$ images are similar and show a compact and round appearance.

PNG 352.1-00.0. - This source was detected by IRAC in all the four bands (Figure 24). The $\mathrm{H} \alpha$ image shows an arcuate nebula around a star. Much more extended structures are revealed by the IRAC images which show a sharp edge to the east and a diffuse tail trailing toward the west. Based on its morphology, it is likely to be an H II region and not a $\mathrm{PN}$. The cometary structure shown by the MIR images is commonly seen in compact and ultracompact $\mathrm{H}$ II regions and implies the interaction between the nebula and the ISM. We also find that the object is surrounded by very extended ( 10 arcmin across $)$ fainter filaments. The $24 \mu \mathrm{m}$ emission is dominated by the central bright region and appears as a seemingly oval structure. This object is an example showing that these MIR data can help for identifying/rejecting PNs. We note that there are two bright IR sources, IRAS 17221 - 3533 and 2MASS J17253388 - 3536005 (the latter might be a YSO; Felli et al. 2002), lying within the nebulosity.

PNG 352.8-00.5. - This source was detected by IRAC in all the four bands (Figure 25). It has a IRAS counterpart, IRAS $17262-3511$ and is classified as a 'True' PN in the MASH II catalogue. The NVSS survey gives a flux density of $72.8 \pm 2.3 \mathrm{mJy}$ at 1.4 GHz (Condon et al. 1998). We note that the PN lies within an overlap region between the GLIMPSE I and GLIMPSE II surveys and its IRAC images have already investigated by Ramos-Larios \& Phillips (2008). We refer the reader to their paper for further details of this 
PN. Its $24 \mu \mathrm{m}$ emission is very strong and saturates the detector. The SED shows a smooth increase of flux from $1-50 \mu \mathrm{m}$, and suggests the presence of cold dust with $T=60-100 \mathrm{~K}$ (Figure 44).

PNG 353.9+00.0. - This source was detected by IRAC in all the four bands (Figure 26). Optical spectroscopy suggests a low excitation class and the object is classified as a 'Possible' PN by Parker et al. (2006). The H $\alpha$ and MIR images appear as a point source. Unlike the other PNs, this source has a IRAC color only slightly redder than stars, and has a low SED slope $\left(\alpha_{\text {IRAC }}=-0.91\right)$. The SED indicates to a color temperature of about $1000 \mathrm{~K}$ (Figure 44), or even higher if the interstellar extinction is non-negligible. This value is significantly higher than in normal PNs. Consequently, this object is more likely to be an emission-line star rather than a PN.

PNG 355.6-01.4. - This source is almost overwhelmed by the bright background emission and was detected by IRAC in the $8.0 \mu \mathrm{m}$ band only (Figure 27). It is classified as a 'True' PN by Parker et al. (2006). The NVSS survey gives a flux density of $5.5 \pm 0.6 \mathrm{mJy}$ at $1.4 \mathrm{GHz}$ (Condon et al. 1998). The $8 \mu \mathrm{m}$ image is visually consistent with the $\mathrm{H} \alpha$ appearance that shows a small bipolar-like structure.

PNG 355.6+01.4. - This source was detected by IRAC in all the four bands (Figure 28). It is classified as a 'Likely' PN by Parker et al. (2006). The spectroscopy suggests that it probably is a very low excitation PN. The NVSS survey gives a flux density of $8.2 \pm 0.6 \mathrm{mJy}$ at $1.4 \mathrm{GHz}$ (Condon et al. 1998). The $\mathrm{H} \alpha$ and MIR images appear as a point source. The SED implies the presence of cold dust with $T<100 \mathrm{~K}$ (Figure 44).

PNG 356.0-01.4. - This source was detected by IRAC in the 5.6 and $8.0 \mu \mathrm{m}$ bands only (Figure 29). It is classified as a 'True' PN by Parker et al. (2006). The NVSS survey gives a flux density of $4.8 \pm 0.5 \mathrm{mJy}$ at $1.4 \mathrm{GHz}$ (Condon et al. 1998). The $\mathrm{H} \alpha$ and IRAC images show that it is a faint, compact, and elliptical PN.

PNG 356.9+00.9. - This source was detected by IRAC in all the four bands (Figure 30 ). It is classified as a 'True' PN by Parker et al. (2006). The NVSS survey gives a flux density of $12.5 \pm 0.6 \mathrm{mJy}$ at $1.4 \mathrm{GHz}$ (Condon et al. 1998). The $\mathrm{H} \alpha$ image appears as a point source. However, extended emission is clearly resolved in the IRAC images which exhibit a round structure. The PN is very red and bright in the MIR bands. The SED implies the presence of cold dust with $T<150 \mathrm{~K}$ (Figure 44).

PNG 357.4-01.3. - This source was detected by IRAC in all the four bands (Figure 31). Given its large size ( $\sim 5$ arcmin $)$, the object is unique in our sample. Parker et al. (2001) suggested that it might be a highly evolved PN. As the extended emission region is very diffuse, we cannot obtain reliable integrated flux and can only roughly estimate the size. 
The optical image shows that it is a ring nebula around the Wolf-Rayet (WR) star WR 101. Cappa et al. (2002) reported the radio image of this nebula at $1465 \mathrm{MHz}$ taken through the VLA and obtained an ionized mass of about $230 \mathrm{M}_{\odot}$. Therefore, this object is unlikely to be a PN, and is probably a WR nebula consisting of mass lost as well as interstellar material swept-up by the stellar wind from an evolved massive star. We find that the $\mathrm{H} \alpha$ and $24 \mu \mathrm{m}$ images have a high resemblance. However, compared to that in $\mathrm{H} \alpha$ and $24 \mu \mathrm{m}$, the ring structure is less well defined and fuzzier in the IRAC bands, which also reveal more diffuse emission extending to the northwest. The position of the bright clump in the southeast as shown by IRAC slightly differs from that of the $\mathrm{H} \alpha$ counterpart which appears to be closer to the ionized source. This suggests that the nebulosity is optically thick to the ionizing photons, and probably the dust grains close to the ionized source have been destructed by photodissociation of the central star.

PNG 357.5+01.3. - This source was detected by IRAC in all the four bands (Figure 32). It is classified as a 'Likely' PN by Parker et al. (2006). The $\mathrm{H} \alpha$ image appears as a point source. Extended emission is clearly resolved in the IRAC images, which show that it is a red and round nebula.

PNG 357.7+01.4.- This source was detected by IRAC in all the four bands (Figure 33). It is classified as a 'True' PN by Parker et al. (2006). The $\mathrm{H} \alpha$ image shows a slightly elongated structure. The IRAC images only reveal a bright core and the object is the most compact PN in our sample.

PNG 358.2-01.1.- This source was detected by IRAC in all the four bands (Figure 34). This PN has been discovered before (e.g. Cahn \& Kaler 1971; Acker et al. 1991). The NVSS survey gives a flux density of $<2.5 \mathrm{mJy}$ at $1.4 \mathrm{GHz}$ (Condon et al. 1998). Using the energybalance method, Preite-Martinez et al. (1989) obtained the temperature of the central star to be $144,000 \mathrm{~K}$. Maciel (1984) determined a distance toward this PN of $1.1 \mathrm{kpc}$. It is described by Parker et al. (2001) as a bright, compact, possibly bipolar PN with arc features. The IRAC images have a visually similar appearance with the $\mathrm{H} \alpha$ image and show arc features and fuzzy extended structure.

PNG 358.8-00.0.- This source was detected by IRAC in all the four bands (Figure 35). The NVSS survey gives a flux density of $<2.5 \mathrm{mJy}$ at $1.4 \mathrm{GHz}$ (Condon et al. 1998). Although the object has a IRAS counterpart, IRAS 17395 - 2950, we cannot find the flux measurements in the IRAS PSC. Volk et al. (1991) presented the low-resolution IRAS spectrum and classified the PN into Group I which represents noisy or incomplete spectra. The $\mathrm{H} \alpha$ image shows a small and fuzzy nebula. It has a large $\alpha_{\text {IRAC }}$ value, and is redder than all the other GLIMPSE II PNs. The MIR images are remarkably extended and reveal intriguing structure. The bright region has a C-shape. We find that it has a large dust envelope with 
a cavity centered on the central star and diffuse emission extending to the west. Thus it is unlikely to be a PN. The SED implies the presence of cold dust with $T<150 \mathrm{~K}$ (Figure 44). From the IRAC images, we can also see a bipolar nebulosity in the northwest to PNG 358.8-00.0. This object has no visible optical counterpart and is probably associated with a compact radio source discovered by Gray et al. (1993). From the morphology, we conjecture that it is likely to be a PN or compact H II region highly obscured by dust extinction. Follow-up observations of this GLIMPSE-survey-discovered object are required to unveil its nature.

PNG 359.1-01.7. - This source was detected by IRAC in all the four bands (Figure 36). This is a previously known PN (e.g. Henize et al. 1967; Webster 1975). A detailed optical spectrophotometric study has been recently presented by Wang \& Liu (2007). The deduced chemical abundances suggest that it a Type I PN. The NVSS survey gives a flux density of $91.9 \pm 2.8 \mathrm{mJy}$ at $1.4 \mathrm{GHz}$ (Condon et al. 1998). Zhang (1995) derived a distance to this PN of $3.30 \mathrm{kpc}$. Using a dynamical method, Gesicki \& Zijlstra (2007) obtained the mass of the central star of $\sim 0.627 \mathrm{M}_{\odot}$. The IRAC and $\mathrm{H} \alpha$ images show a compact, bright, and round appearance. However, Gesicki et al. (2003) found that this object has complicate line profiles of H I, [N II], and [O III], and suggested the presence a bipolar outflow. In the IRAC images, we do note that the brightest part is not located in the center of the nebula. The SED indicates to a color temperature $T<300 \mathrm{~K}$ (Figure 44).

PNG 359.2+01.2. - This source was detected by IRAC in all the four bands (Figure 37). It has been known as a typical bipolar PN (e.g. Kohoutek 1982; Ratag et al. 1990; Corradi \& Schwarz 1995) and has been suggested as a symbiotic system (Corradi 1995). The NVSS survey gives a flux density of $13.5 \pm 0.6 \mathrm{mJy}$ at $1.4 \mathrm{GHz}$ (Condon et al. 1998). It has a IRAS counterpart, IRAS $17358-2854$. The $\mathrm{H} \alpha$ image shows a bright core with a highly collimated bipolar outflow. The compact core has also been detected by Lee et al. (2007) at radio wavelengths. The IRAC images show a less extreme bipolar shape with a lower major/minor axis ratio compared to the $\mathrm{H} \alpha$ image. We speculate that the $\mathrm{PN}$ has a central dust torus therefore enhancing the IRAC emission near the center. Interestingly, the appearance revealed by the $24 \mu \mathrm{m}$ image is a bipolar nebulosity surrounded by an extended halo with a size of $30^{\prime \prime} \times 42^{\prime \prime}$. The SED indicates to the presence of cold dust with $T<100 \mathrm{~K}$ (Figure 44).

PNG 359.3-00.9. - This source was detected by IRAC in all the four bands (Figure 38). This is a well-known bipolar nebula $\mathrm{Hb} 5$ and has been extensively studied (see, e.g. Pottasch \& Surendiranath 2007; Montez et al. 2009, for recent literature summaries of this object). Optical images show that this PN is composed of two closed-end bipolar lobes, a bright compact core, concentric rings and some filaments. It is a high-excitation Type I 
PN and suffers from heavy extinction. The distance to this PN is not accurately known, with estimates ranging from 1-7 kpc (Montez et al. 2009). It is one of the four PNs that have hitherto been detected with X-ray emission, indicating the presence of shock-heated gas in the bipolar nebula (Montez et al. 2009). The NVSS survey gives a flux density of $179.5 \pm 5.4 \mathrm{mJy}$ at $1.4 \mathrm{GHz}$ (Condon et al. 1998). The IRAC images exhibit the bright compact core, which is so luminous that the $8 \mu \mathrm{m}$ band is saturated. Because of the brightness of the central core, the bipolar lobes are not obvious from the IRAC images. It is clear that in the IRAC wavelengths, the core-to-lobe flux-density ratio is larger than that at $\mathrm{H} \alpha$. We suggest that the $8 \mu \mathrm{m}$ emission is dominated by the dust thermal emission from a central torus. The $24 \mu \mathrm{m}$ band, however, shows more extended emission even when deconvolved using a point source function. The $24-\mu \mathrm{m}$ image suggests an extended cold dust envelope, corresponding to the $\sim 100 \mathrm{~K}$ dust component seen in the SED (Figure 44). The ISO spectrum of $\mathrm{Hb} 5$ is plotted on the SED, showing that ISO flux measurements are in good agreement with other measurements. We note that there are two strong high-ionization lines $[\mathrm{Ne} \mathrm{V}]$ $24 \mu \mathrm{m}$ and [O IV] $26 \mu \mathrm{m}$ lying in the $24 \mu \mathrm{m}$ band, which contribute about 10 percent of the total $24 \mu \mathrm{m}$ flux and are partly responsible for the saturation of the central region in the $24 \mu \mathrm{m}$ image.

\subsection{Nondetections}

The following MASH PNs have no obvious IRAC counterparts: PNG 000.0-01.8, PNG 000.1+01.9, PNG 000.6 - 01.4, PNG 001.0 - 01.4, PNG 001.1-01.2 PNG 001.2+01.3, PNG 001.5-01.6, PNG 001.6+00.1, PNG 001.6-00.6, PNG 001.6+01.6, PNG 001.9+01.9, PNG 002.1+01.2, PNG 002.4+01.1, PNG 002.5+01.3, PNG 002.7-01.4, PNG 004.0-00.4, PNG 004.8-00.5, PNG 005.5-00.8, PNG 007.2+00.0, PNG 009.9-00.2, PNG 354.0-00.8, PNG $354.3+00.5$, PNG 354.8-00.5, PNG 355.5-01.1, PNG 355.9+00.7, PNG 357.3+01.3, PNG 357.6+01.0, PNG 359.1-00.7, PNG 359.2-01.2, PNG 359.2+01.3, PNG 359.3+01.4, PNG 359.5-01.8, PNG 359.9-01.8, PNG 359.9+01.8. In most of the cases, the detection of these PNs is hampered by the bright background near the Galactic center. For several objects (e.g. PNG 001.1 - 01.2 and PNG 002.7 - 01.4), the IRAC bands exhibit apparent point sources near the corresponding positions, but without extended emission around these sources. They are likely to be field stars although some of them have a rising flux distribution from 3.8 to $8.0 \mu \mathrm{m}$. An interesting object is PNG $354.8-00.5$, which is a radio and maser source. Its $\mathrm{H} \alpha$ image reveals a faint, circular nebula with a size of $42.0^{\prime \prime} \times 37.0^{\prime \prime}$. However, the IRAC images show an extremely luminous star, which is bright enough to saturate the detector and makes the nebula 'invisible'. The object probably represent a evolutionary stage of PN formation around a very evolved AGB star, as suggested by Cohen et al. (2005b). 


\section{DISCUSSION}

All the objects studied in our sample can be categorized as ionized nebulae with cold dust emission. The ionized nature of the objects is confirmed by $\mathrm{H} \alpha$ and radio continuum emission. The peak of the dust continuum mostly lies between 10 and $30 \mu \mathrm{m}$, suggesting dust temperatures of 100-300 K. Since the infrared spectra of PNs are rich in ionic emission lines as well as solid-state emission bands (see, e.g., Stanghellini et al. 2007), accurate determination of dust temperatures of PNs is very difficult without spectra and our estimates represent only rough approximations.

\subsection{Implications for Source Sizes at Different Wavelengths}

It is instructive to compare the morphologies observed in different bands since they could trace the spatial distributions of different nebular components. Figure 49 shows histograms of source sizes in MIR wavelengths relative to $\mathrm{H} \alpha$, for which we have deconvolved the point spread functions (PSF) simply through $\theta_{\mathrm{s}}=\sqrt{\theta_{\text {obs }}^{2}-\theta_{\text {res }}^{2}}$ (note that this is only a rough estimate as the PSFs are non-Gaussian), where $\theta_{\mathrm{s}}$ is the intrinsic source sizes, $\theta_{\mathrm{obs}}$ is the observed sizes, and $\theta_{\text {res }}$ is the angular resolution. An inspection of this figure shows that (1) the $\mathrm{H} \alpha$ sizes are more similar to those observed in 5.8 and $8.0 \mu \mathrm{m}$ compared to the other MIR sizes, (2) the $24 \mu \mathrm{m}$ sizes are generally larger than those in the other wavelengths, and (3) The 3.6 and $4.5 \mu \mathrm{m}$ sizes are quite similar to each other and are more compact than those in the other bands.

The optical and $8.0 \mu \mathrm{m}$ morphologies of a certain PN can be remarkably different. For some PNs, the $8.0 \mu \mathrm{m}$ emission has a larger angular extent than the corresponding optical image. This is indicative of the presence of extended dust envelopes which obscure the optical emission and emit thermal continuum and AIB features contributing to the $8.0 \mu \mathrm{m}$ band. This can also in part be attributed to the $\mathrm{H}_{2}$ rotational-vibrational transitions excited by shocks. On the other hand, some PNs are more compact at $8 \mu \mathrm{m}$ than at $\mathrm{H} \alpha$. An important issue is whether this really reflects the different spatial distributions of the different components rather than different instrumental sensitivities. In order to investigate this problem, in Figure. 50 we compare the $\mathrm{H} \alpha$ and $8.0 \mu \mathrm{m}$ flux profiles along the major axis of the bipolar PN PNG 359.2+01.2. While the $\mathrm{H} \alpha$ and $8.0 \mu \mathrm{m}$ images have comparable angular resolutions, it is clear that the $8.0 \mu \mathrm{m}$ emission is more centrally concentrated than the $\mathrm{H} \alpha$ emission. The most likely interpretation is that the $\mathrm{H} \alpha$ and $8.0 \mu \mathrm{m}$ emissions originate from different regions, the former from the bipolar lobes and the latter from a nuclear dust torus. Examples of infrared-observed equatorial dust torus can be found in the bipolar proto-PN IRAS $17441-2411$ (Volk et al. 2007). If we generalize from this example, objects with 
smaller angular extents in $8.0 \mu \mathrm{m}$ than in $\mathrm{H} \alpha$ may be bipolar nebulae.

The $24 \mu \mathrm{m}$ images of our sample objects are in general well resolved, extended, and show a circular appearance with a sharp boundary. It is unlikely that [Ne V] $24.3 \mu \mathrm{m}$ and [O IV] $25.9 \mu \mathrm{m}$ lines are dominant contributor to the $24 \mu \mathrm{m}$ flux otherwise the $24 \mu \mathrm{m}$ image would show a more compact appearance than the $\mathrm{H} \alpha$ image. It is more probable that the $24 \mu \mathrm{m}$ images correspond to the distribution of cold dust which is the remnant of AGB mass loss. Extended AGB halos have been frequently detected in deep optical images of PNs (Corradi et al. 2003). If the cold dust grains are plentifully present within these halos, they can exhibit strong $24 \mu \mathrm{m}$ emission.

For high-excitation PNs, the $[\mathrm{Ne} \mathrm{V}]$ and [O IV] line emission may significantly enhance the $24 \mu \mathrm{m}$ emission in nebular centers. A recent study of MIPS $24 \mu \mathrm{m}$ images of Galactic PNs (which are generally more extended than objects in our sample) has been reported by Chu et al. (2009), who find that the sizes and surface brightnesses of $\mathrm{H} \alpha$ and $24 \mu \mathrm{m}$ emission suggest an evolutionary sequence.

From Figures 39 43, we can see some of our sample objects show faint and extended halos and ring structures around their bright cores. While some of these are clearly instrumental artifacts, some are likely to be real (e.g., PNG 001.0 - 01.9, PNG 001.0 + 01.9, PNG 359.1 01.7 etc.) and deserve further study. A recent MIPS24-based study to search for debris disk around stars has found $24 \mu \mathrm{m}$ excess emission in the Helix Nebula and suggested that it originates from a dust disk at a distance of 40-100 AU from the central star, and that the dust is produced by collisions of Kuiper-Belt-like objects ( $\mathrm{Su}$ et al. 2007). Assuming that the distances to the GLIMPSE II PNs are typically larger than $1 \mathrm{kpc}$, we find that the $24 \mu \mathrm{m}$ emission extends to $>5000 \mathrm{AU}$ from the center, more than a factor of 100 times farther than the Sun's Kuiper Belt, and thus is unlikely to arise from a Kuiper Belt around lowand intermediate-mass AGB stars. However, we cannot completely rule out the possibility that Kuiper Belt-like objects may partly contribute to the $24 \mu \mathrm{m}$ emission in the centers. An interesting PN is PNG $001.0+01.9$ which is the only object showing more compact $24 \mu \mathrm{m}$ emission relative to $\mathrm{H} \alpha$ in our sample. Its $24 \mu \mathrm{m}$ emission is probably in part associated with the central star.

For the two short-wavelengths IRAC bands $(3.6$ and $4.5 \mu \mathrm{m})$, the contributions from the hydrogen recombination line and bound-free emission should not be dominant, because if so, the 3.6 and $4.5 \mu \mathrm{m}$ emission would morphologically match the $\mathrm{H} \alpha$ emission well. The spatially compact 3.6 and $4.5 \mu \mathrm{m}$ emission probably suggest significant contribution from photospheric emission. This conclusion is further supported by the SEDs (Figure 44) and color-color diagrams (Figures 46] and 477). 


\subsection{IRAC $8.0 \mu \mathrm{m}$ versus $M S X 8.3 \mu \mathrm{m}$ Integrated Fluxes}

Reach et al. (2005) investigated the absolute calibration of IRAC. They found that the extended emission surface brightness is incorrectly calibrated and should be multiplied by an effective aperture correction factor ( 0.737 for the $8.0 \mu \mathrm{m}$ band). This was subsequently established by Cohen et al. (2007a) that the IRAC $8.0 \mu \mathrm{m}$ surface brightness should be scaled down by a factor of $0.74 \pm 0.07$ based on comparison between the IRAC $8.0 \mu \mathrm{m}$ and $M S X$ $8.3 \mu \mathrm{m}$ fluxes of a sample of $43 \mathrm{H}$ II regions. This study predicts a IRAC8.0/MSX8.3 flux ratio of $1.14 \pm 0.02$ for diffuse $\mathrm{H}$ II regions based on the different contributions from AIB emission in the two different bandpasses. A similar study of 14 PNs (Cohen et al. 2007b) also confirm their previous results. Here we present an independent examination of the IRAC $8.0 \mu \mathrm{m}$ calibration using the integrated fluxes of the GLIMPSE II PNs which are very compact diffuse sources.

A total of 10 GLIMPSE II PNs have $M S X 8.3 \mu \mathrm{m}$ detections. Figure 51 compared the spatially integrated fluxes detected in the IRAC $8.0 \mu \mathrm{m}$ and $M S X 8.3 \mu \mathrm{m}$ bands. For comparison, we also overplot the values for GLIMPSE I PNs. A good correlation between the two fluxes is clearly shown. The PN with the largest IRAC/MSX flux discrepancy is PNG 003.5 + 01.3, which has a large $M S X 8.3 \mu \mathrm{m}$ flux compared with the IRAC $8.0 \mu \mathrm{m}$ flux. Combining the GLIMPSE I and GLIMPSE II measurements, we deduce an average IRAC8.0/MSX8.3 flux ratio of $0.90 \pm 0.36$, consistent with the median value of $1.2 \pm 0.2$ found by Cohen et al. (2007b) for PNs but slightly lower than that of $1.55 \pm 0.15$ obtained by Cohen et al. (2007a) for H II regions.

Cohen et al. (2007b) found a marginal relation between the IRAC8.0/MSX8.3 flux ratio and nebular diameters which suggests that the aperture correction factor increases from 1.0 at small nebular size (i.e. point-source) to $\geq 1.4$ at $77^{\prime \prime}$. This relation is not shown in Figure 51 because our sample does not include very extended PNs. The PNs investigated here have a diameter smaller than $60^{\prime \prime}$, and mostly smaller than $20^{\prime \prime}$, much more compact than the arcminutes-scale H II regions. Therefore, we infer that the absolute flux calibration for the compact sources studied here should be accurate, and we do not need to make aperture correction to obtain the integrated fluxes of the GLIMPSE II PNs.

The [S IV] $10.5 \mu \mathrm{m}$ line, which can contribute to the $M S X 8.3 \mu \mathrm{m}$ band but not to the IRAC $8.0 \mu \mathrm{m}$ band, is partly responsible to the IRAC8.0/MSX8.3 flux ratio. PNs often have higher excitation class than H II regions, and thus are supposed to have stronger [S IV] emission. As a result, the IRAC8.0/MSX8.3 flux ratio in PNs (especially high-excitation $\mathrm{PNs}$ ) might be lower compared with that in $\mathrm{H}$ II regions.

We cannot completely rule out variability as the partial cause of the IRAC and MSX flux 
difference for individual PNs since the GLIMPSE survey was performed about ten years after the $M S X$ observations. If this is the case, our study will have implications to understand the pulsations mechanism or binary system in PNs. Another possible reason causing the IRAC/MSX flux discrepancy in individual PNs is that the $M S X$ fluxes of some PNs might be contaminated by blended sources since $M S X$ has a resolution approximate ten times lower than IRAC.

\subsection{MIPS $24 \mu \mathrm{m}$ versus $M S X 21 \mu \mathrm{m}$ Integrated Fluxes}

The ratio of MIPS $24 \mu \mathrm{m}$ to $M S X 21 \mu \mathrm{m}$ fluxes provides an opportunity to establish the reliability of absolute diffuse calibration and our measurements of MIPS $24 \mu \mathrm{m}$ fluxes. For this purpose, one should at first estimate the predicted MIPS24/MSX21 flux ratio through

integrating the spectral response functions to the SEDs. Cohen (2009) investigated the diffuse absolute calibration of the MIPS $24 \mu \mathrm{m}$ channel using a sample of $\mathrm{H}$ II regions, and obtained the observed MIPS24/MSX21 flux ratio of $1.19 \pm 0.03$. Comparing with the predicted value of $1.18 \pm 0.02$, which is obtained from three SEDs pertinent to H II regions, they concluded that the absolute diffuse calibration of MIPS24 is consistent with that of $M S X 21$ within $3 \%(1 \sigma)$. We reasonably suppose that predicted MIPS24/MSX21 flux ratio of PNs is nearly the same as that of H II regions.

In Figure 52 we compare the MIPS $24 \mu \mathrm{m}$ and $M S X 21 \mu \mathrm{m}$ integrated fluxes of the GLIMPSE II PNs. The sensitivity of the MSX21 band is approximately 1000 times lower than of the MIPS24 band, and thus only relatively bright PNs can be detected by MSX21. We found that nine of the GLIMPSE II PNs have MSX21 counterparts. Since the MIPS24 band has a much lower saturated level, very bright sources in the $M S X$ catalogue could be saturated in MIPS. This is clearly reflected in Figure 52. Excluding the two saturated sources, we obtain the MIPS24/MSX21 flux ratio of $1.19 \pm 0.12$, in perfect agreement with value for H II regions (Cohen 2009). We note that the H II regions investigated by Cohen (2009) are much more extended, and generally have $24 \mu \mathrm{m}$ integrated fluxes ranging from $10^{4.0}-10^{5.5} \mathrm{mJy}$, significantly larger than those of the GLIMPSE II PNs in Figure 52 (about $\left.10^{3.2}-10^{3.9} \mathrm{mJy}\right)$. The consistent results obtained from PNs and H II regions, which have remarkably different spatial sizes and integrated fluxes, strongly validate the reliabilities of the absolute diffuse calibration of MIPS24 and our flux measurements. 


\subsection{Comparison with Radio Flux Densities}

A tight correlation between far- and mid-infrared and radio emission from star-forming galaxies has been established and has been a subject of intense discussion for many years (see, e.g., Appleton et al. 2004, and the references therein). The origin of the tightness of this correlation is, however, not completely understood. It would be interesting to test the $\mathrm{MIR} /$ radio relation in PNs, although they have quite different physical conditions from star formation regions.

If the MIR emission is dominated by dust heated by ultraviolet (UV) photons from the central stars which also ionize the gaseous nebulae and are responsible for free-free thermal emission in the radio continuum, we would see a correlation between the MIR and radio emission in PNs. Cohen et al. (2007b) compared the IRAC $8.0 \mu \mathrm{m}, M S X 8.3 \mu \mathrm{m}$, and radio flux densities and obtained an overall MIR/radio ratio of $4.6 \pm 1.2$. They also suggest that, unlike that in $\mathrm{H}$ II regions, the $\mathrm{MIR}$ /radio ratio in PNs might change with $\mathrm{PN}$ evolution as the result of decreasing amount of UV photons available to pump the AIB into MIR fluorescence. Even without considering the line emission factor, the dust continuum flux of PNs will decrease as the dust cools due to geometric dilution of the heating photons, which will occur at a different rate as the radio continuum flux which decreases as the electron density drops as the result of expansion even the electron temperature remains constant.

We note that all the GLIMPSE II PNs are located within the NVSS $1.4 \mathrm{GHz}$ survey (Condon et al. 1998) field, and 23 of them have NVSS detections. They have sizes smaller than the $1.4 \mathrm{GHz}$ beam size of VLA and are spatially unresolved at this frequency. In Figure 53 we compare the IRAC $8.0 \mu \mathrm{m}$ and MIPS $24 \mu \mathrm{m}$ integrated fluxes with those from NVSS catalogue. It is evident that albeit with a large dispersion, there is a positive correlation between the MIR and radio emission. The correlation coefficients in the logarithmic space are 0.66 for IRAC $8.0 \mu \mathrm{m}$ vs NVSS $1.4 \mathrm{GHz}$, and 0.64 for MIPS $24 \mu \mathrm{m}$ vs NVSS $1.4 \mathrm{GHz}$. Figure 53 also shows that the number distributions of the IRAC8.0 $\mu \mathrm{m} / 1.4 \mathrm{GHz}$ and MIPS2 $4 \mu \mathrm{m} / 1.4 \mathrm{GHz}$ flux ratios are peaked at $\sim 3$ and $\sim 100$, respectively. If we assume that these GLIMPSE II PNs are approximately at the same evolutionary stage and that the non-thermal emission is insignificant, the dispersion in Figure 53 should be due to self-absorption of radio emission. For most compact PNs, the turnover (from optically thick to optically thin) frequency is usually at $1-5 \mathrm{GHz}$, so it is quite possible that some of these nebulae are optically thick at $1.4 \mathrm{GHz}$. The object showing the largest MIR/radio flux ratio is PNG 359.2+ 01.2 which has a high density torus.

A basic observable parameter $q_{\mathrm{IR}}\left[=\log \left(S_{\mathrm{IR}} / S_{1.4 \mathrm{GHz}}\right), S\right.$ being the flux density] is often used to characterize the MIR/radio relation. For instance, Appleton et al. (2004) obtained $q_{24}=1.00 \pm 0.27$ for galaxies with different redshifts; Ibar et al. (2008) found $q_{24}=0.71 \pm 0.47$ 
for a sample at high-redshifts; Wu et al. (2008) obtained the $q_{24}$ values for dwarf star-forming galaxies generally ranging from 0.9 to 1.3 with one extreme case of 2.2. For the GLIMPSE II PNs, we obtain $q_{8.0}=0.79 \pm 0.52$ and $q_{24}=2.13 \pm 0.37$. The $q_{8.0}$ value is consistent with that found for PNs in the Magellanic Clouds by Filipović et al. (2009). The larger $q_{24}$ value in PNs compared to those in star-forming galaxies is a reflection of the fact that PNs and galaxies have different radio and infrared emitting mechanisms. Galaxies radiate nonthermal radiation whereas PNs radiate thermal f-f emission. The dust components in PNs are heated by a single central star whereas the dust in galaxies is heated primarily by massive stars.

\section{CONCLUSIONS}

An investigation of the MASH PNs within the GLIMPSE II field has revealed that 37 optically identified PNs have MIR counterparts. These PNs are distinguishable from the surrounding stars by their redder color and more extended angular sizes. From their infrared morphologies, we suggest that some of these objects (PNG 352.1-00.0, PNG 353.9+00.0, PNG 357.4-01.3, PNG 358.8-00.0) are unlikely to be PNs. We present the spatially integrated flux and size measurements of these PNs at 3.6, 4.5, 5.8, 8.0, and $24 \mu \mathrm{m}$. MIR color-color and color-magnitude diagrams are produced. Combining our results with those in other data archives, we construct the $0.82-100 \mu \mathrm{m}$ SEDs for these objects. The primary findings from analysis of these results are summarized below.

1. Compared to the GLIMPSE I PNs, the GLIMPSE II PNs are generally more compact and have lower spatially integrated fluxes, implying that these PNs towards the Galactic bulge might be generally more distant. Nevertheless, the MIR colors of GLIMPSE I and II PNs are very similar to each other, and are clearly different with those of stellar objects.

2. Despite some GLIMPSE II PNs are invisible in the $3.6-5.8 \mu \mathrm{m}$ bands and are only marginally above the IRAC $8.0 \mu \mathrm{m}$ detection limitation, all of them are characterized with very strong $24 \mu \mathrm{m}$ emission, which is likely to originate from extended cold dust envelopes.

3. The nebular morphologies and fluxes detected in different wavelengths allow us to separate the different components in the nebulae. The 5.8 and $8.0 \mu \mathrm{m}$ emission may be either more or less compact than their optical counterparts, respectively implying the presence of dust toruses centered on bipolar PNs or extended neutral envelopes around ionized gaseous nebulae. 
4. From the anomalous colors of some objects, we infer the contributions of AIB emissions to the IRAC fluxes.

5. The absolute diffuse calibrations are examined by comparing the IRAC $8.0 \mu \mathrm{m}$ and MIPS $24 \mu \mathrm{m}$ to the $M S X 8.3$ and $21 \mu \mathrm{m}$ fluxes. We obtain the IRAC8.0/MSX8.3 and MIPS24/MSX21 flux ratios of $0.90 \pm 0.36$ and $1.19 \pm 0.12$, respectively. These results are reasonably consistent with previous studies by Cohen et al. (2007a), Cohen et al. (2007b), and Cohen (2009) using data for H II regions and GLIMPSE I PNs, and suggest good calibrations for these slightly resolved GLIMPSE II PNs.

6. We explore the MIR/radio relation for PNs. A loose correlation between the MIR $(8.0 \mu \mathrm{m}$ and $24 \mu \mathrm{m})$ and the NVSS $1.4 \mathrm{GHz}$ fluxes is found, and can be statistically described by $q_{\mathrm{IR}}$ - the ratio between the flux densities at MIR and $1.4 \mathrm{GHz}$. For the GLIMPSE II PNs, we find $q_{8.0}=0.79 \pm 0.52$ and $q_{24}=2.13 \pm 0.37$. The larger $q_{\mathrm{IR}}$ values and the larger dispersion in the MIR/radio correlation of PNs compared to those of star-forming galaxies are attributed to their respective different underlying radiation mechanisms.

Since the bulk of GLIMPSE II PNs are located at higher Galactic latitudes $\left(1^{\circ} \leq|b| \leq\right.$ $2^{\circ}$ ), we anticipate that a large number of PNs might have been detected in the GLIMPSE 3D legacy survey, which extends the latitude coverage to $\pm 3^{\circ}$. The study of GLIMPSE 3D PNs will be presented in a subsequent paper.

We thank Ed Churchwell and the GLIMPSE team for assistance in the processing and analysis of GLIMPSE survey data. We also thank Nico Koning for his help in data processing and Jun-ichi Nakashima for fruitful discussion. This work is based on observations made with the Spitzer Space Telescope, which is operated by the Jet Propulsion Laboratory, California Institute of Technology, under a contract with NASA. This study used data products from the GLIMPSE and MIPSGAL Spitzer Space Telescope Legacy Programs. This publication also used the data products from the DENIS project, which has been partly funded by the SCIENCE and the HCM plans of the European Commission under grants CT920791 and CT940627, from the Two Micron All Sky Survey, which is a joint project of the University of Massachusetts and the Infrared Processing and Analysis Center/California Institute of Technology, funded by the National Aeronautics and Space Administration and the National Science Foundation, and from the Midcourse Space Experiment, which was funded by the Ballistic Missile Defense Organization with additional support from NASA Office of Space Science. This research has also made use of the NASA/IPAC Infrared Science Archive, which is operated by the Jet Propulsion Laboratory, California Institute of Technology, 
under contract with the National Aeronautics and Space Administration, and made use of the SIMBAD data base, operated at CDS, Strasbourg, France. Support for this work was provided by the Research Grants Council of the Hong Kong under grants HKU7032/09P.

\section{REFERENCES}

Acker, A., Köppen, J., Stenholm, B., \& Raytchev, B. 1991, A\&AS, 89, 237

Appleton, P. N. et al. 2004, ApJS, 154, 147

Benjamin, R. A., et al. 2003, PASP, 115, 953

Cahn, J. H., \& Kaler, J. B., 1971, ApJS, 22, 319

Cappa, C. E., Goss, W. M. \& Pineault, S. 2002, ApJ, 123, 3348

Carey, S. J. et al. 2008, MIPSGAL v3.0 Data Delivery Description Document (29 August 2008)

Chavarría, L. A., Allen, L. E., Hora, J. L., Christopher, M. B., \& Fazio, G. G. 2008, ApJ, 682,445

Chu, Y.-H. et al. 2009, AJ, 138, 691

Churchwell, E. et al. 2007, ApJ, 670, 428

Churchwell, E. et al. 2009, PASP, 121, 213

Cohen, M. 2009, AJ, 137, 3449

Cohen, M. et al. 2005a, ApJ, 627, 446

Cohen, M., Parker, Q. A., \& Chapman, J. 2005b, MNRAS, 357, 1189

Cohen, M. et al. 2007a, MNRAS, 374, 979

Cohen, M. et al. 2007b, ApJ, 669, 343

Condon, J. J., \& Kaplan, D. L. 1998, ApJS, 117, 361

Corradi, R. L. M., Schönberner, D., Steffen, M., \& Perinotto, M. 2003, MNRAS, 340, 417

Corradi, R. L. M., \& Schwarz, H. E. 1995, MNRAS, 293, 871 
Corradi, R. L. M. 1995, MNRAS, 276, 521

Cox, P. et al. 2002, A\&A, 384, 603

de la Fuente, E. et al. 2008, astro-ph/0812.3446

Exter, K. M., Barlow, M. J., \& Walton, N. A. 2004, MNRAS, 349, 1291

Fazio et al. 2004, ApJS, 154, 10

Felli, M., Testi, L., Schuller, F. \& Omont, A. 2002, A\&A, 392, 971

Filipović et al. 2009, MNRAS, 399, 769

Gesicki, K., Acker, A., \& Zijlstra, A. A. 2003, A\&A, 400, 957

Gesicki, K., \& Zijlstra, A. A. 2007, A\&A, 467, L29

Gray, A. D., Whiteoak, B. Z., Cram, L. E., \& Goss, W. M. 1993, MNRAS, 264, 678

Henize, K. G. 1967, ApJS, 14125

Hora, J. L., Latter, W. B., Allen, L. E., Marengo, M., Deutsch, L. K., \& Pipher, J. L. 2004, ApJS, 154, 296

Hora, J. L., et al. 2008, ApJ, 135, 726

Ibar, E. et al. 2008, MNRAS, 386, 953

Jacoby, G. H., \& Van de Steene, G. C. 2004, A\&A, 419, 563

Kistiakowsky, V., \& Helfand, D. J. 1995, AJ, 110, 2225

Kohoutek, L. 1982, A\&A, 115, 420

Kohoutek, L. 1994, Astron, Nachr., 315, 235

Kohoutek, L. 2002, Astron, Nachr., 323, 57

Kwok, S. 1982, ApJ, 258, 280

Kwok, S. 1990, MNRAS, 244, 179

Kwok, S., Zhang, Y., Koning, N., Huang, H.-H., \& Churchwell, E. 2008, ApJS, 174, 426 (Paper I)

Lada, C. J. et al. 2006, ApJ, 131, 1574 
Lee, T.-H., Lim, J., \& Kwok, S. 2007, ApJ, 665, 341

Maciel, W. J. 1984, A\&AS, 55, 253

Meade, M. R. et al. 2007, GLIMPSE3D v1.0 Data Release (23 August, 2007)

Meade, M. R. et al. 2008, GLIMPSEII v2.0 Data Release (18 April, 2008)

Montez, R. J., Kastner, J. H., Balick, B., \& Frank, A. 2009, ApJ, 694, 1481

Miszalski, B., Parker, Q. A., Acker, A., Birkby, J. L., Frew, D. J., \& Kovacevic, A. 2008, MNRAS, 384, 525

Parker, Q.A., Phillipps, S., Morgan, D.H. 1999, in Taylor, A.R., Landecker, T.L., Joncas, G., eds. ASP Conf. Ser. Vol. 168, New Perspectives on the Interstellar Medium. Astron. Soc. Pac., San Francisco, p. 126

Parker, Q. A. et al. 2001, The Edinburgh/AAO/Strasbourg Catalogue of Galactic Planetary Nebulae

Parker, Q. A. et al. 2006, MNRAS, 373, 79

Phillips, J. P. \& Ramos-Larios, G. 2008a, MNRAS, 383, 1029

Phillips, J. P. \& Ramos-Larios, G. 2008b, MNRAS, 386, 995

Pottasch, S. R. \& Surendiranath, R. 2007, A\&A, 462, 179

Pottasch, S.R., Baud, B., Beintema, D., Emerson, J., Habing, H.J., Harris, S., Houck, J., Jennings, R., Marsden, P. 1984, A\&A, 138, 10

Preite-Martinez, A., Acker, A., Köppen, J., \& Stenholm, B. 1989, A\&AS, 81, 309

Ramos-Larios, G. \& Phillips, J. P. 2008, MNRAS, 390, 1014

Ratag, M. A., Pottasch, S. R., Zijlstra, A. A., \& Menzies, J. 1990, A\&A, 233, 181

Reach, W. T. et al. 2005, PASP, 117, 978

Rieke, G. H. et al. 2004, ApJS, 154, 25

Schwarz, H. E., Corradi, R. L. M. \& Melnick, J. 1992, A\&AS, 96, 23

Stanghellini, L. et al. 2007, ApJ, 671, 1669

Su, K. Y. L. et al. 2004, ApJS, 153, 302 
Su, K. Y. L. et al. 2007, ApJ, 657, L41

Ueta, T. 2006, ApJ, 650, 228

Van de Steene, G. C. \& Jacoby, G. H. 2001, A\&A, 373, 536

Volk, K., Kwok, S., Stencel, R. E., \& Brugel, E. 1991, ApJS, 77, 607

Volk, K., Kwok, S., \& Hrivnak, B. J. 2007, ApJ, 670, 1137

Wang, W., \& Liu, X.-W. 2007, MNRAS, 381, 669

Webster, B. L. 1975, MNRAS, 173, 437

Wu, Y., Charmandaris, V., Houck, J.R., Bernard-Salas, J., Lebouteiller, V., Brandl, B.R., Farrah, D. 2008, ApJ, 676, 970

Zhang, C. Y. 1995, ApJS, 98, 659

Zhang, C.Y., \& Kwok, S. 1991, A\&A, 250, 179 


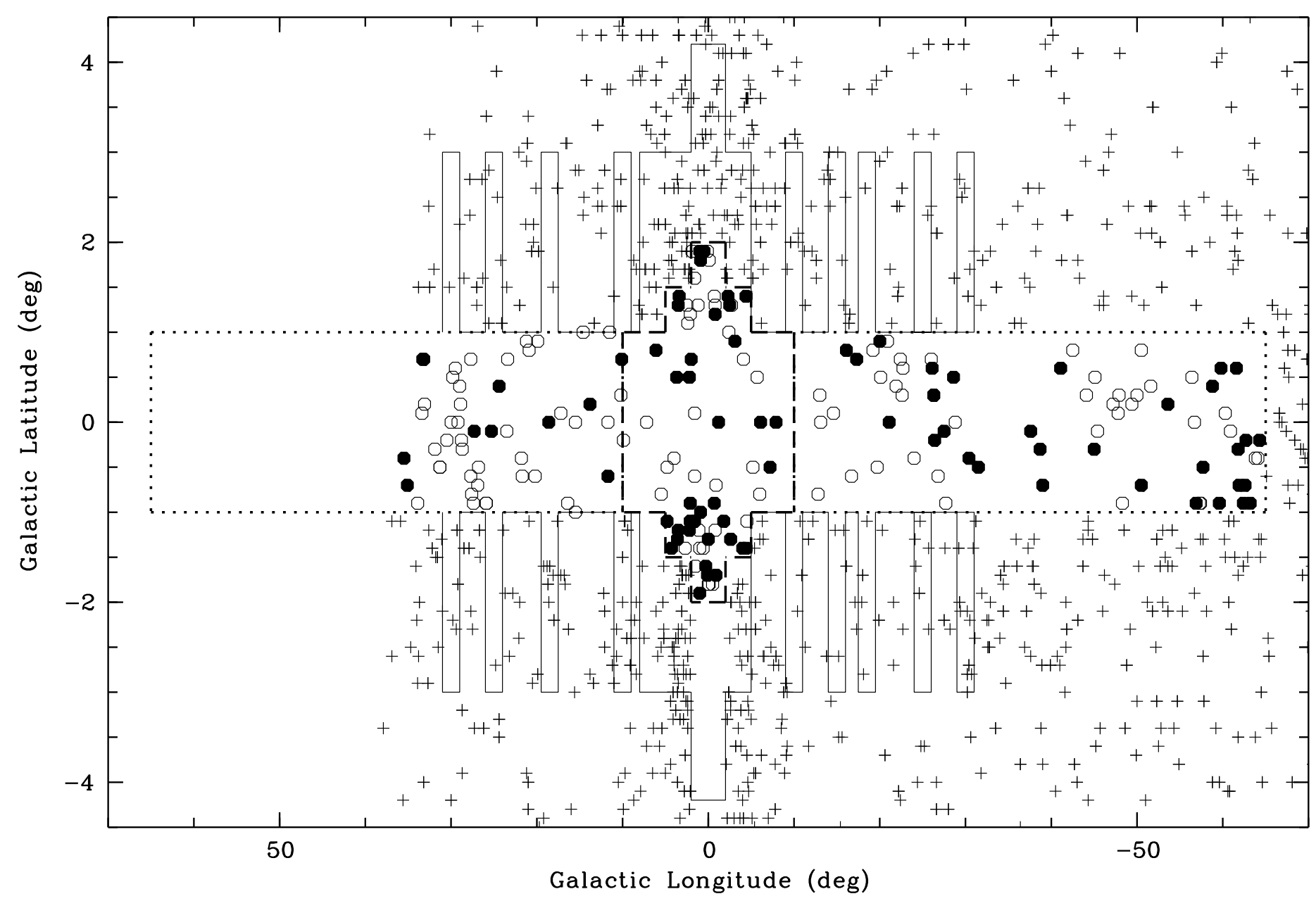

Fig. 1.- The survey coverage of GLIMPSE I (dotted lines), GLIMPSE II (dotted-dashed lines), and GLIMPSE 3D (solid lines). The circles and pluses represent the MASH PNs inside and outside the GLIMPSE I/II survey coverage, respectively. The filled circles denote the MASH PNs having IRAC counterparts that are presented in this paper and Paper I. 
Fig. 2.- Composite-color images of PNG 000.0 - 01.3 made from 3 IRAC bands. The $3.6 \mu \mathrm{m}$ is shown as blue, the $5.8 \mu \mathrm{m}$ is shown as green, and the $8.0 \mu \mathrm{m}$ is shown as red. The lower panel is the same with the upper panel but overlaid with the contours of $\mathrm{H} \alpha$ emission. The target is put in the center of each panel. The coordinates are in units of R.A. (J2000.0 on the horizontal scale) and decl. (J2000.0 on the vertical scale).

Fig. 3.- As in Figure. 2, but for PNG 000.1 - 01.7.

Fig. 4.- As in Figure. 2, but for PNG 000.3 - 01.6.

Fig. 5.- As in Figure. 2, but for PNG 000.5+ 01.9.

Fig. 6.- As in Figure. 2, but for PNG 000.9 - 01.0.

Fig. 7.- As in Figure. 2, but for PNG 000.9+01.8.

Fig. 8.- As in Figure. 2, but for PNG 001.0 - 01.9.

Fig. 9.- As in Figure. 2, but for PNG 001.0 + 01.9.

Fig. 10.- As in Figure. 2, but for PNG 001.6 - 01.1.

Fig. 11.- As in Figure. 2, but for PNG 002.0 + 00.7.

Fig. 12.- As in Figure. 2, but for PNG 002.1 - 00.9.

Fig. 13.- As in Figure. 2, but for PNG 002.1 - 01.1.

Fig. 14. - As in Figure. 2, but for PNG 002.2 + 00.5.

Fig. 15.- As in Figure. 2, but for PNG 002.2 - 01.2.

Fig. 16. - As in Figure. 2, but for PNG 003.4+01.4.

Fig. 17.- As in Figure. 2, but for PNG 003.5 - 01.2.

Fig. 18. - As in Figure. 2, but for PNG 003.5 + 01.3. 
Fig. 19.- As in Figure. 2, but for PNG 003.6 - 01.3.

Fig. 20.- As in Figure. 2, but for PNG 003.7+ 00.5.

Fig. 21.- As in Figure. 2, but for PNG 004.3 - 01.4.

Fig. 22.- As in Figure. 2, but for PNG 004.8 - 01.1.

Fig. 23.- As in Figure. 2, but for PNG 006.1+00.8.

Fig. 24.- As in Figure. 2, but for PNG 352.1 - 00.0.

Fig. 25.- As in Figure. 2, but for PNG 352.8 - 00.5.

Fig. 26. - As in Figure. 2, but for PNG 353.9+ 00.0.

Fig. 27.- As in Figure. 2, but for PNG 355.6 - 01.4.

Fig. 28.- As in Figure. 2, but for PNG 355.6 + 01.4.

Fig. 29.- As in Figure. 2, but for PNG 356.0 - 01.4.

Fig. 30.- As in Figure. 2, but for PNG 356.9+ 00.9.

Fig. 31.- As in Figure. 2, but for PNG 357.4 - 01.3.

Fig. 32.- As in Figure. 2, but for PNG 357.5 + 01.3.

Fig. 33. - As in Figure. 2, but for PNG 357.7+ 01.4.

Fig. 34.- As in Figure. 2, but for PNG 358.2 - 01.1

Fig. 35.- As in Figure. 2, but for PNG 358.8 - 00.0.

Fig. 36. - As in Figure. 2, but for PNG 359.1 - 01.7. 
Fig. 37.- As in Figure. 2, but for PNG 359.2+01.2.

Fig. 38.- As in Figure. 2, but for PNG 359.3 - 00.9.

Fig. 39.- MIPS $24 \mu \mathrm{m}$ images of the GLIMPSE II PNs. From left to right: PNG 000.0-01.3 and PNG 000.1 - 01.7 (1st row); PNG 000.3 - 01.6 and PNG 000.5+01.9 (2nd row); PNG $000.9-01.0$ and PNG 000.9+01.8 (3rd row); PNG 001.0 - 01.9 and PNG 001.0+ 01.9 (4th row). The target is put in the center of each panel. The coordinates are in units of R.A. (J2000.0 on the horizontal scale) and decl. (J2000.0 on the vertical scale).

Fig. 40.- MIPS 24 $\mu \mathrm{m}$ images of the GLIMPSE II PNs. From left to right: PNG 001.6-01.1 and PNG 002.0 + 00.7 (1st row); PNG 002.1 - 00.9 and PNG 002.1 - 01.1 (2nd row); PNG $002.2+00.5$ and PNG 002.2 - 01.2 (3rd row); PNG 003.4+01.4 and PNG 003.5 - 01.2 (4th row). Details are indicated in Figure. 39.

Fig. 41. - MIPS 24 $\mu \mathrm{m}$ images of the GLIMPSE II PNs. From left to right: PNG 003.5+01.3 and PNG 003.6 - 01.3 (1st row); PNG 003.7+00.5 and PNG 004.3-01.4 (2nd row); PNG 004.8 - 01.1 and PNG 006.1 + 00.8 (3rd row), PNG 352.1 - 00.0 and PNG 352.8 - 00.5 (4th row). Details are indicated in Figure. 39.

Fig. 42.- MIPS 24 $\mu \mathrm{m}$ images of the GLIMPSE II PNs. From left to right: PNG 353.9+00.0 and PNG 355.6 - 01.4 (1st row); PNG 355.6+01.4 and PNG 356.0-01.4 (2nd row); PNG $356.9+00.9$ and PNG 357.4 - 01.3 (3rd row); PNG 357.5+01.3 and PNG 357.7+01.4 (4th row). Details are indicated in Figure. 39.

Fig. 43. - MIPS $24 \mu \mathrm{m}$ images of the GLIMPSE II PNs. From left to right: PNG 358.201.1 and PNG 358.8-00.0 (1st row); PNG 359.1-01.7 and PNG 359.2+01.2 (2nd row); PNG 359.3-00.9 (3rd row). Details are indicated in Figure. 39. 

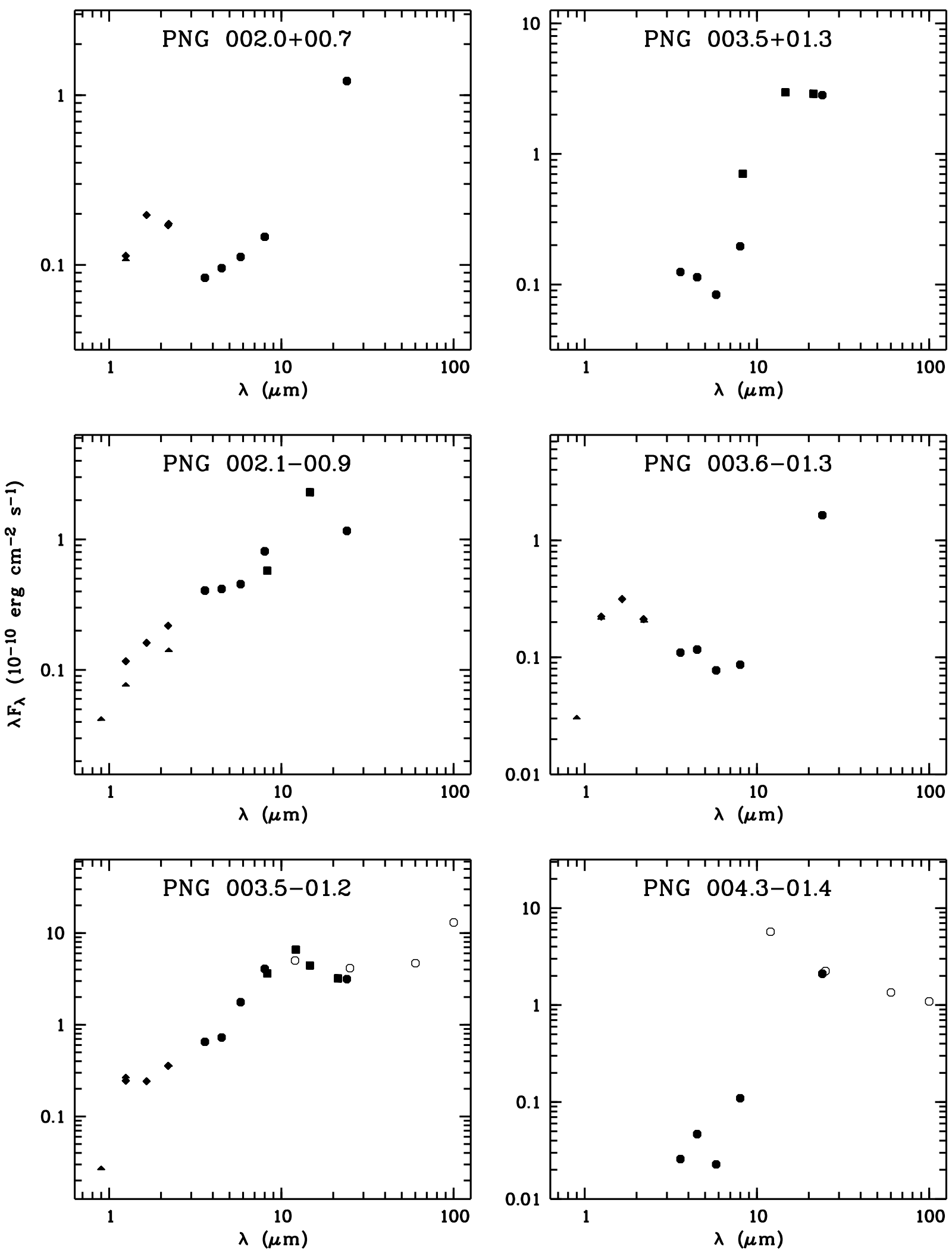

Fig. 44.- The SED of the PNs in the GLIMPSE II sample. The filled triangles, filled diamonds, filled circles, open circles, and filled squares are from the DENIS, 2MASS, GLIMPSE/MIPSGAL, IRAS, and $M S X$ survey, respectively. In the case of PNG 359.3-00.9 

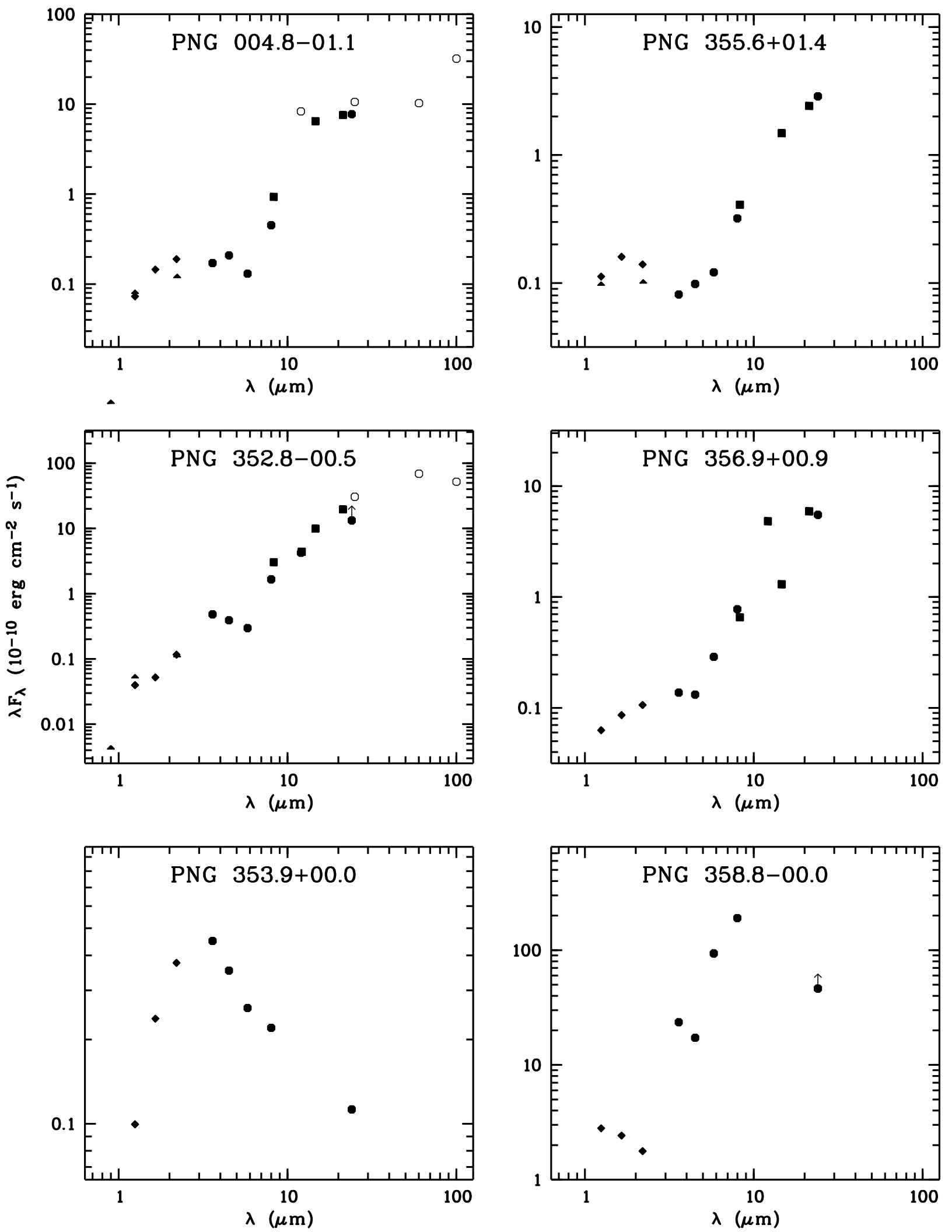

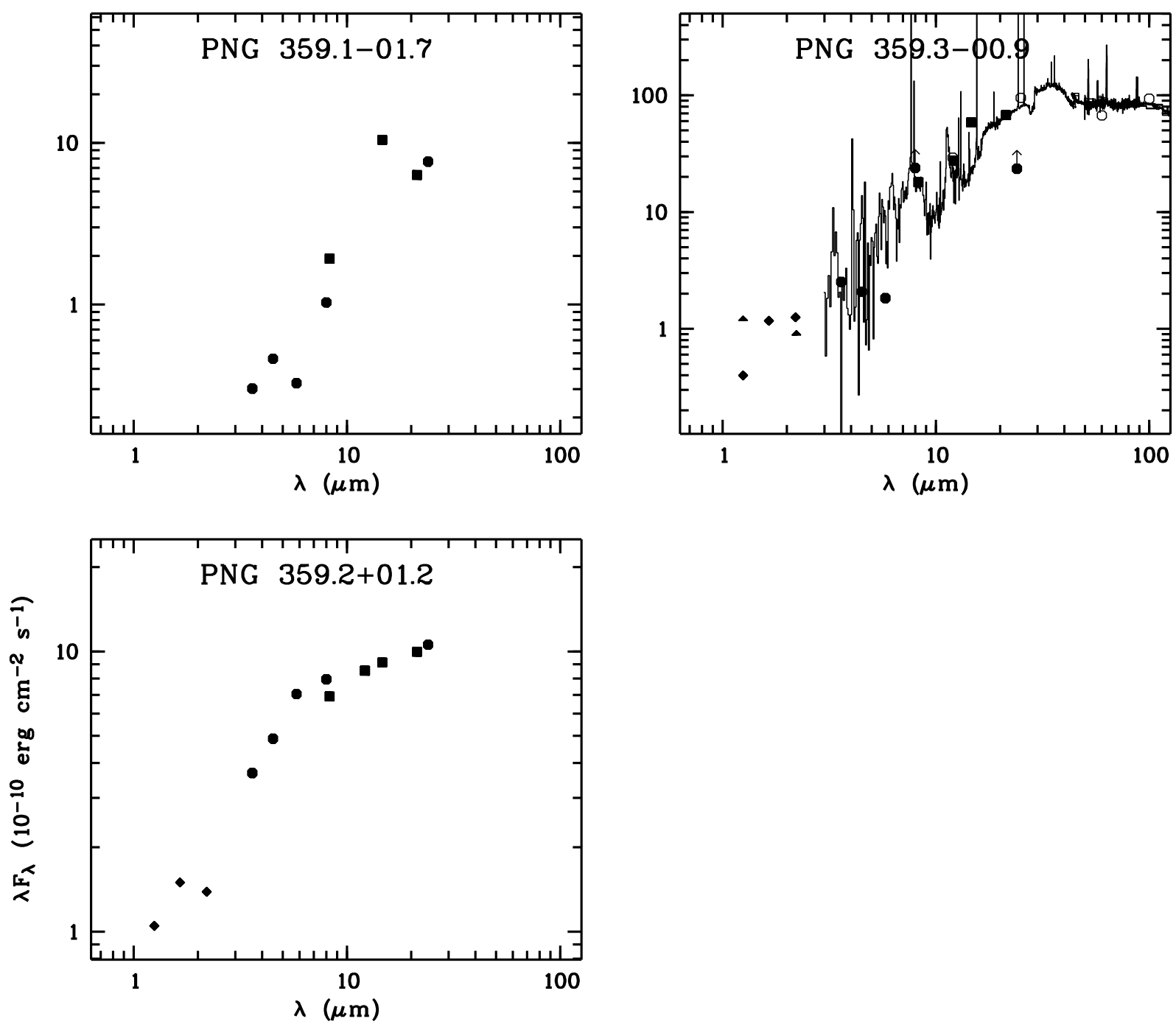


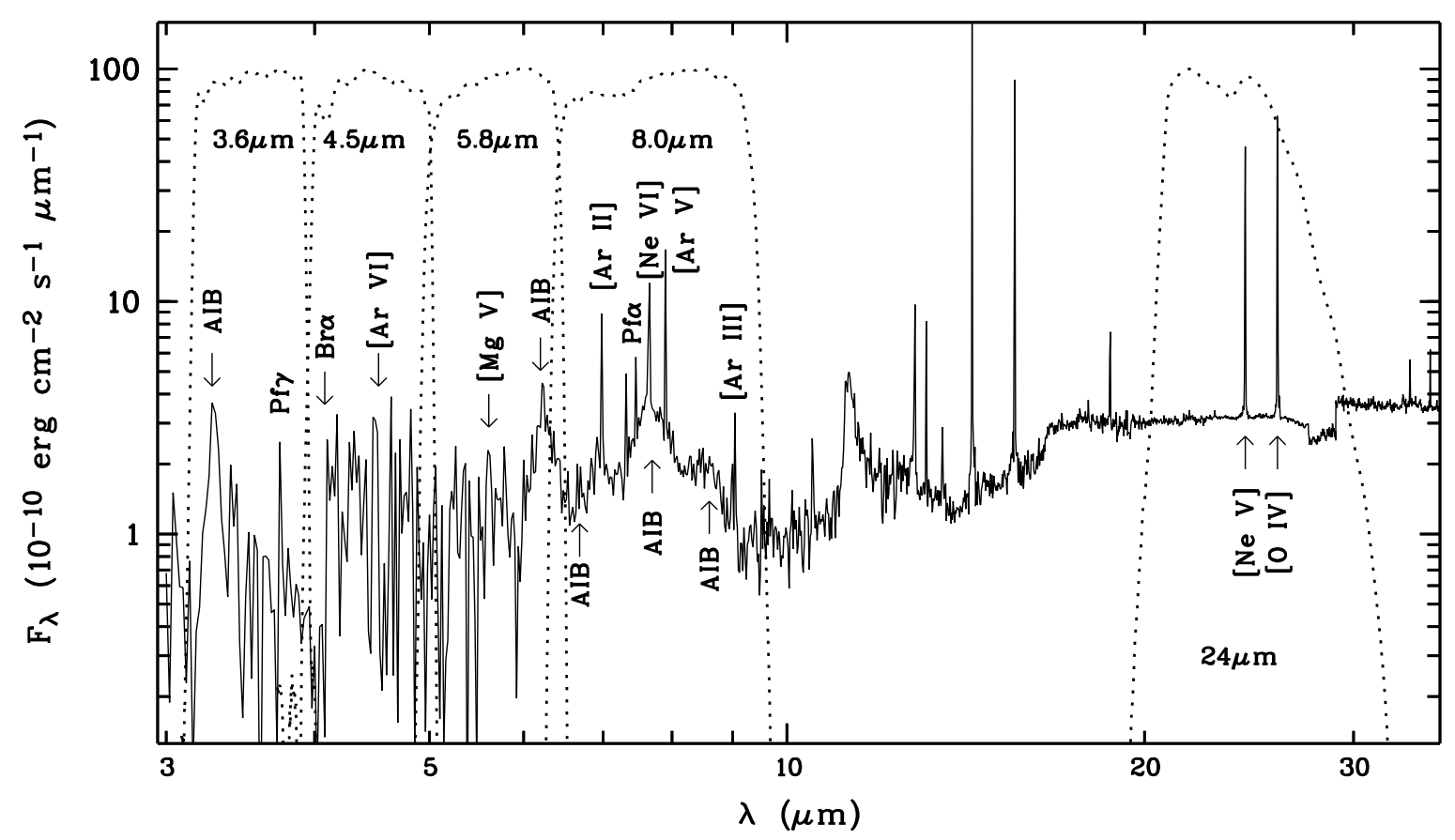

Fig. 45. - The ISO spectrum of $\mathrm{Hb} 5$. The AIB emission and strong atomic lines are identified. The normalized relative spectral response curves for the IRAC and MIPS $24 \mu \mathrm{m}$ bands are overlaid. 


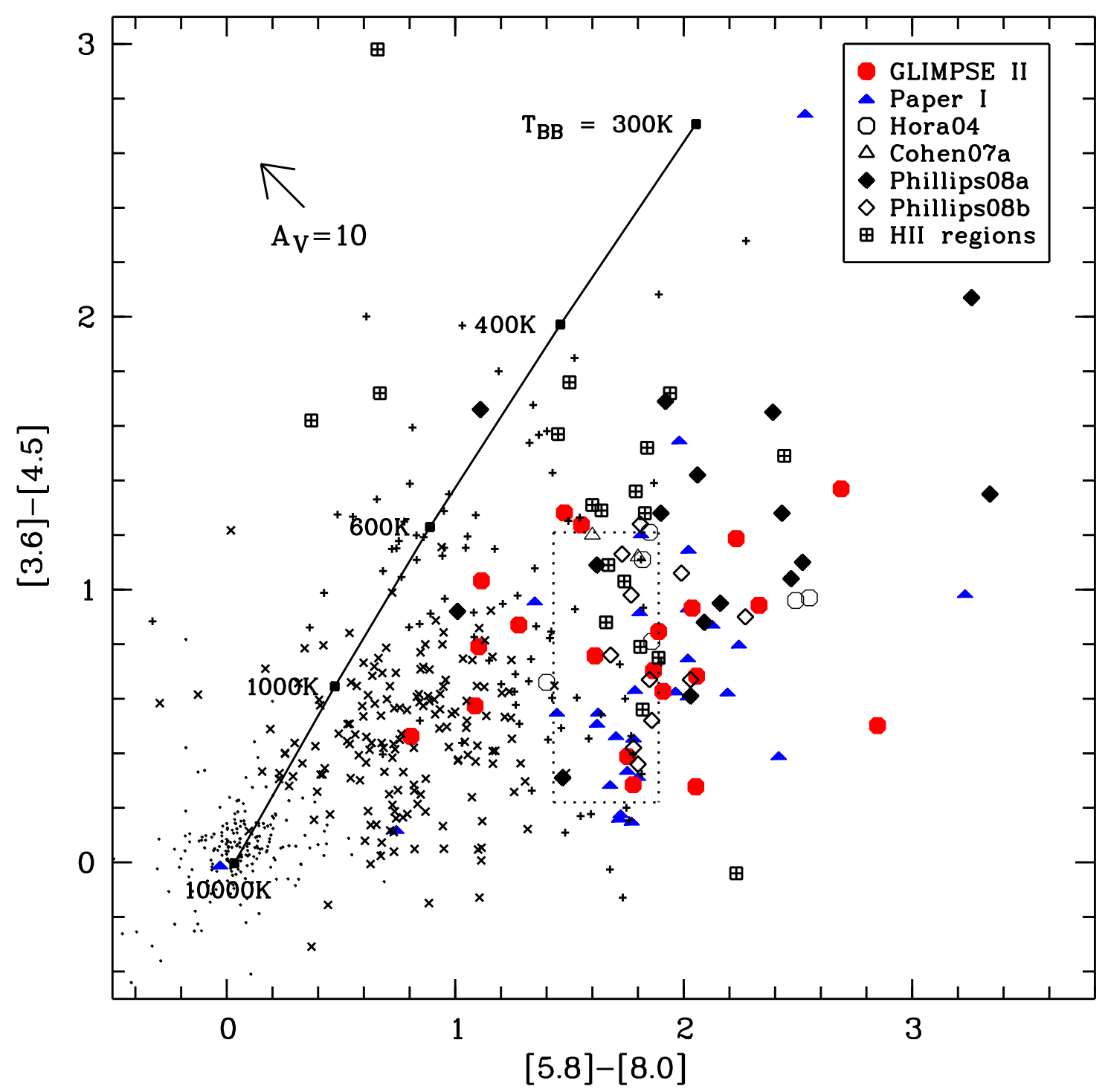

Fig. 46. - IRAC color-color plot ([3.6] - [4.5] vs. [5.8] - [8.0]) for the GLIMPSE I and GLIMPSE II PNs. The observation results by Hora et al. (2004) and Phillips \& Ramos-Larios (2008a, b) and the theoretical calculations by Cohen et al. (2007a) are overlaid. The enclosed zone by dotted lines represents that occupied by the PNs studied by Cohen et al. (2007b). We also show the colors of UC H II regions (de la Fuente et al. 2008) and three types of YSOs (star with infalling envelope - pluses, star with a diskcrosses, and post T-tauri star-points; Chavarría et al. 2008). The filled squares connected by a solid line denote the prediction of blackbody radiation with various temperatures $T_{\mathrm{BB}}$. The arrow in the upper left corner denotes a reddening vector of $A_{v}=10$. 


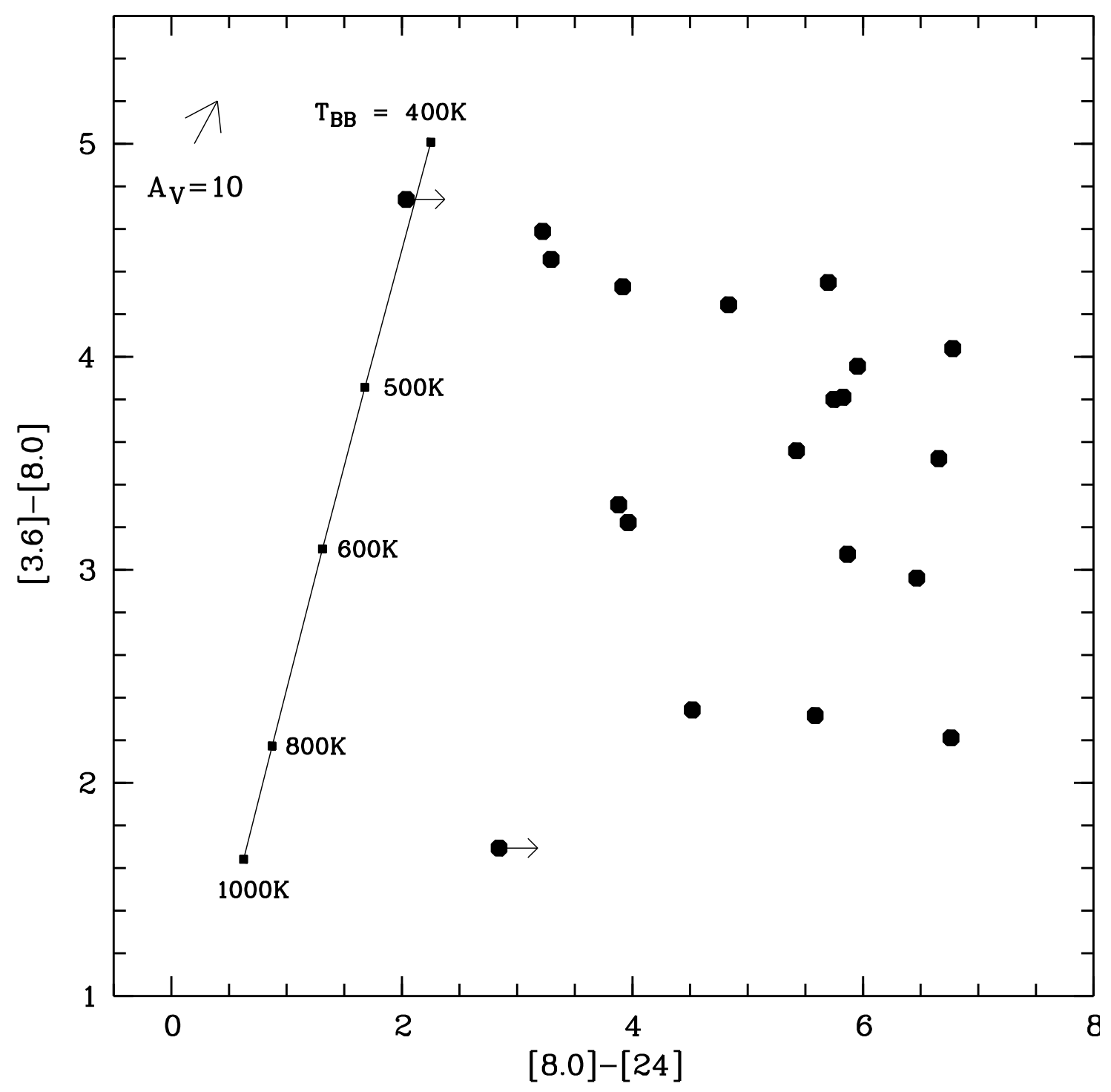

Fig. 47.- The [3.6] - [8.0] versus [8.0] - [24] color-color diagram for the GLIMPSE II PNs. Symbols are otherwise same as in Figure. 46, 


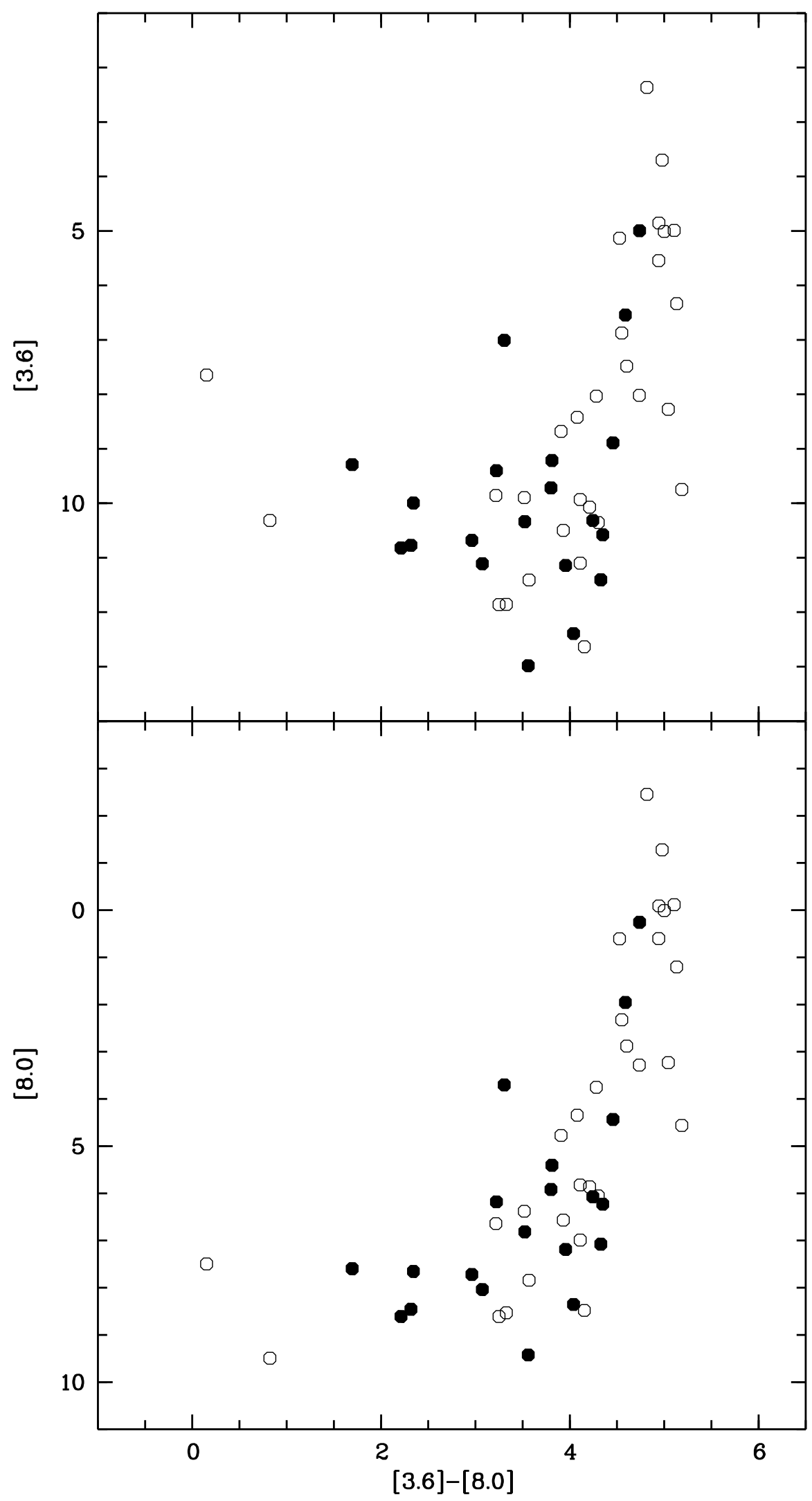

Fig. 48. - The [3.6] versus [3.6] - [8.0] (upper panel) and [8.0] versus [3.6] - [8.0] (lower panel) color-magnitude diagrams for the GLIMPSE I (open circles) and GLIMPSE II (filled circles) PNs. 


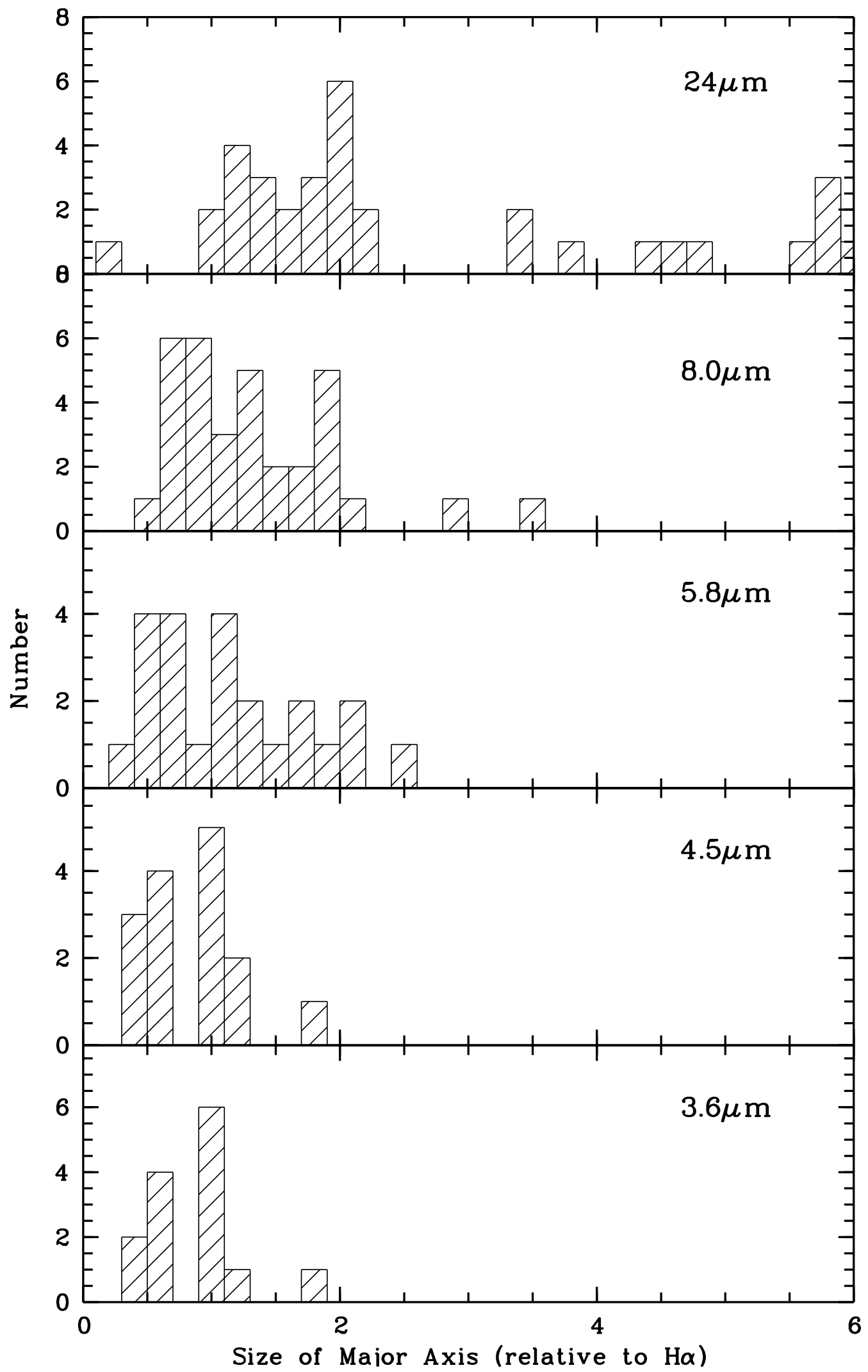

Fig. 49.- The histogram of the relative sizes of nebular major axises measured in different wavelengths. 


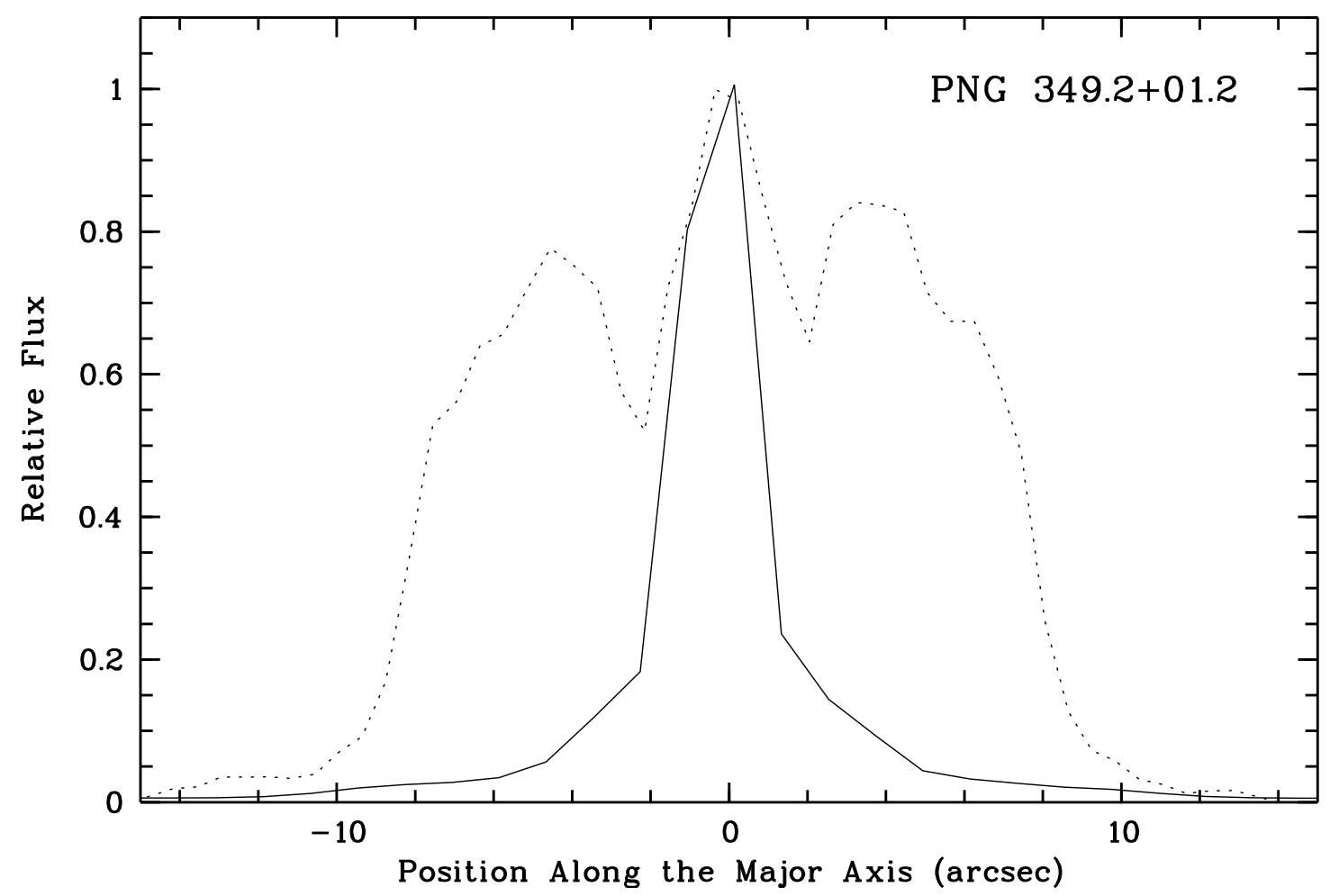

Fig. 50.- The flux distribution along the major axis of the bipolar nebula PNG 359.2+01.2 at $\mathrm{H} \alpha$ (dotted line) and $8.0 \mu \mathrm{m}$ (solid line) bands. 


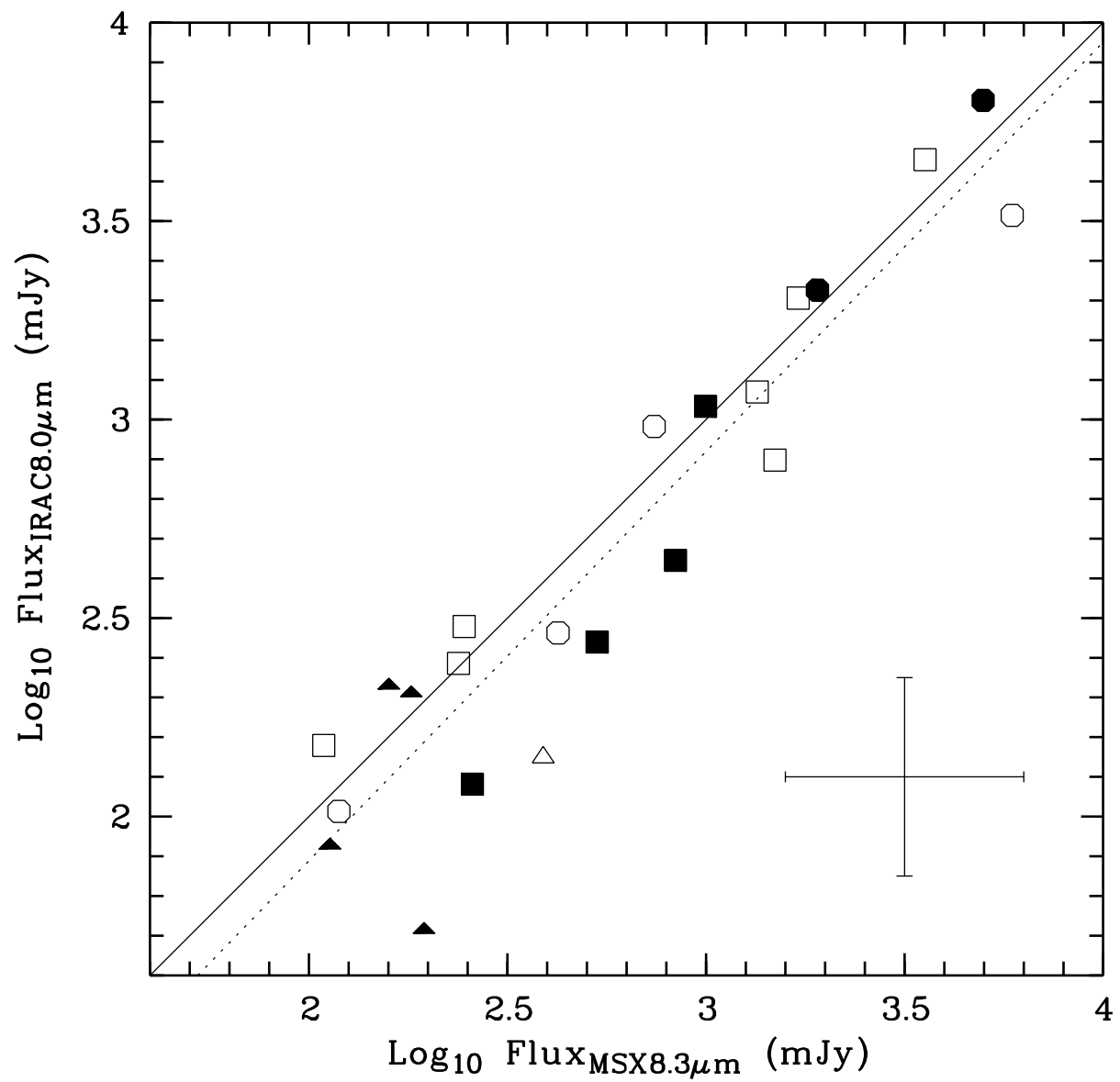

Fig. 51. - IRAC $8.0 \mu \mathrm{m}$ versus MSX $8.3 \mu \mathrm{m}$ integrated fluxes for the GLIMPSE I (open symbols; from Paper I) and GLIMPSE II (filled symbols) PNs. The triangles, squares, and circles denote the PNs with diameters $(R)$ in the ranges of $R \leq 10^{\prime \prime}, 10^{\prime \prime}<R \leq 20^{\prime \prime}$, and $20^{\prime \prime}<R \leq 60^{\prime \prime}$, respectively. The solid diagonal line is a $y=x$ plot. The dotted line represents a linear least-squares fit. The error bar is given in the lower right corner. 


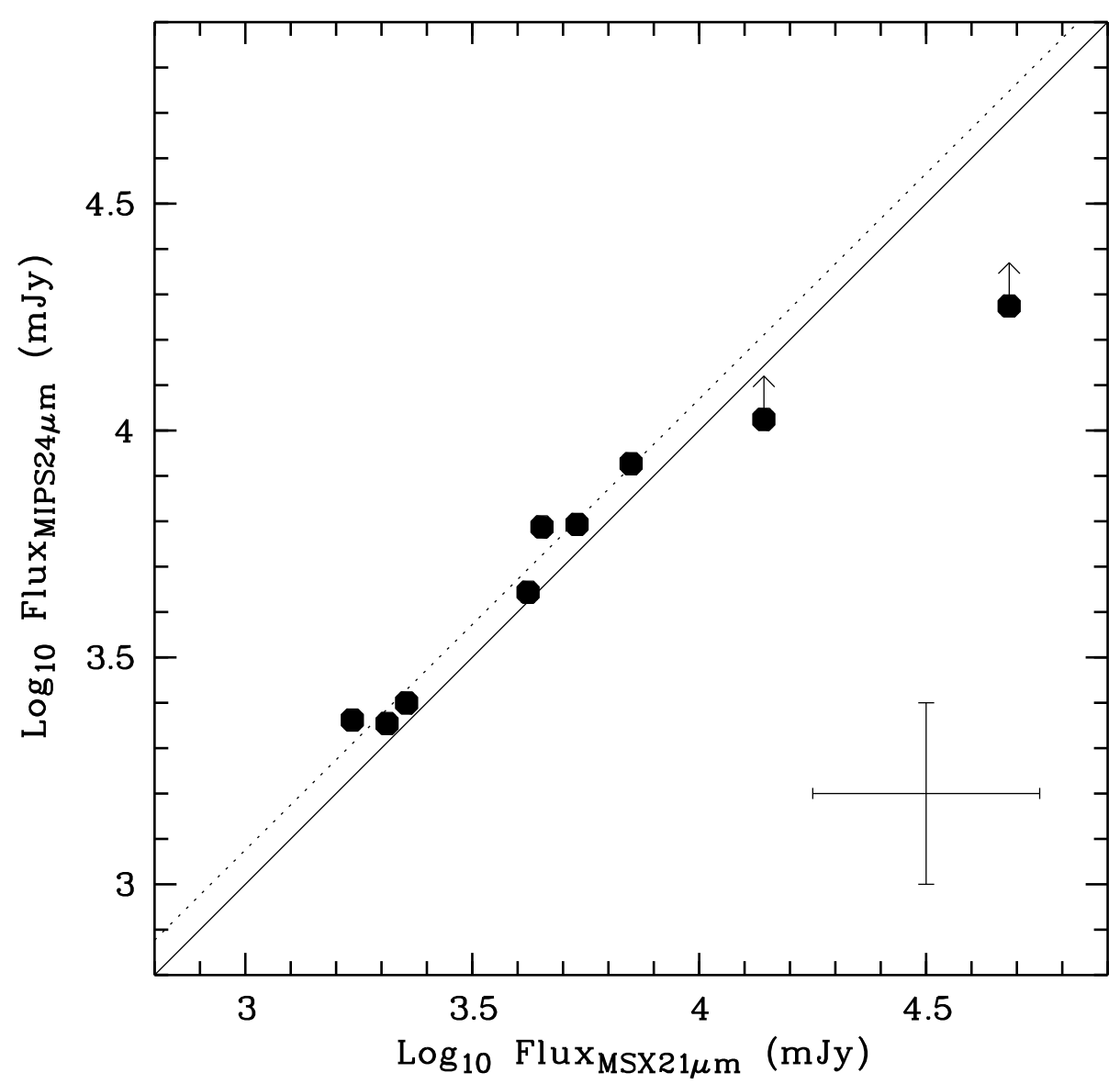

Fig. 52.- IRAC $24 \mu \mathrm{m}$ versus MSX $21 \mu \mathrm{m}$ integrated fluxes for the GLIMPSE II PNs. The solid diagonal line is a $y=x$ plot. The dotted line represents a linear least-squares fit. The error bar is given in the lower right corner. 

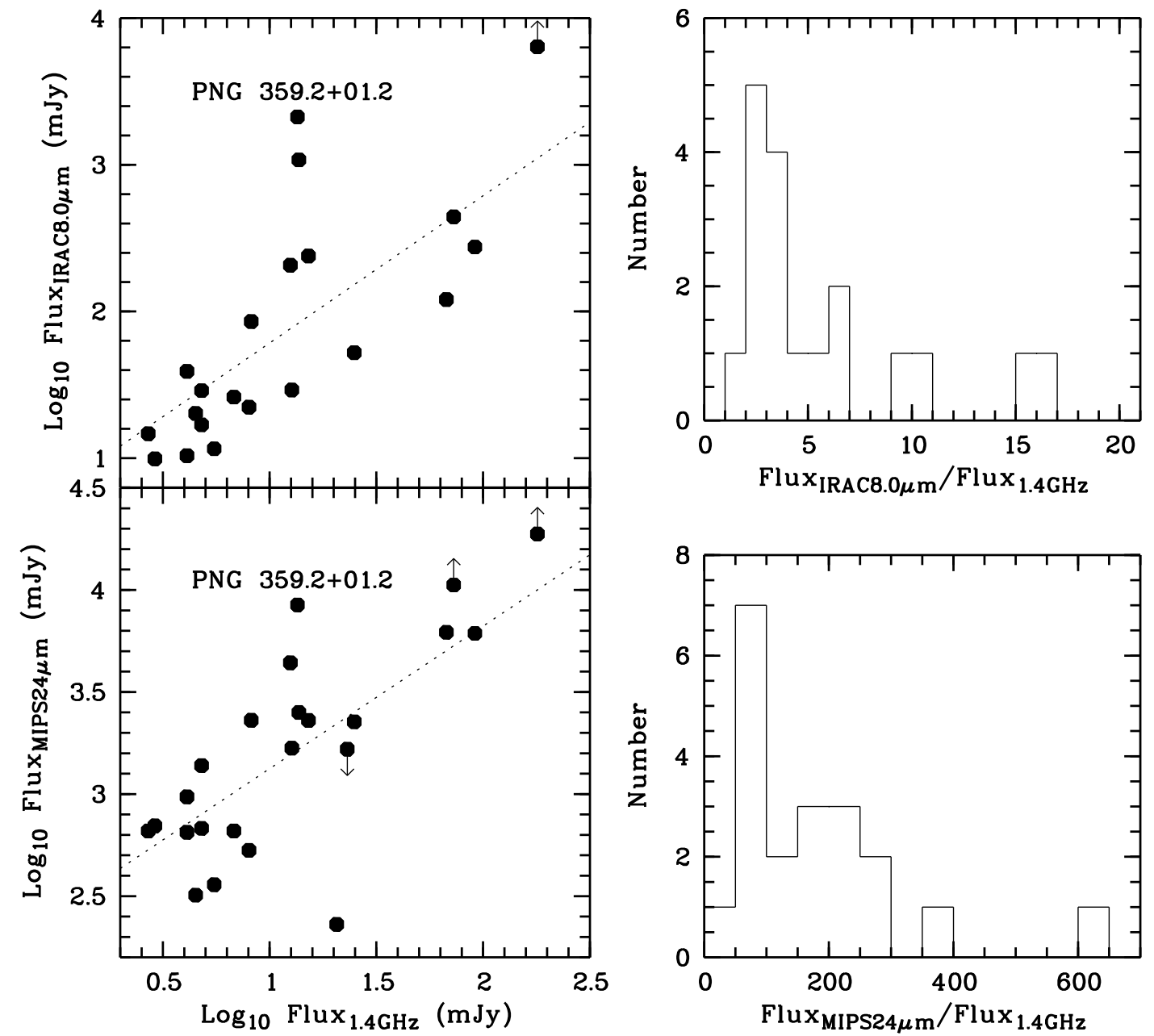

Fig. 53.- Left panels: IRAC $8.0 \mu \mathrm{m}$ (upper panel) and MIPS $24 \mu \mathrm{m}$ (lower panel) versus NVSS 1.4 GHz integrated fluxes for the GLIMPSE II PNs. The dotted lines represent a linear fitting. Right panels: the number distributions of the $8.0 \mu \mathrm{m} / 1.4 \mathrm{GHz}$ (upper panel; note that the two objects with extremely large flux ratios are out of the range of the abscissa) and $24 \mu \mathrm{m} / 1.4 \mathrm{GHz}$ (lower panel) flux ratios. 
Table 1. Known PNs.

\begin{tabular}{cl}
\hline \hline Object & \multicolumn{1}{c}{ Other Name } \\
\hline PNG 000.5 +01.9 & PN K 6-7 \\
PNG 001.0 - 01.9 & PN K 6-35 \\
PNG 001.0 + 01.9 & Hen 2-264, PN K1-4, PN Sa 3-69 \\
PNG 002.2 +00.5 & Terz N 2337 \\
PNG 358.2-01.1 & PN Al 2-L, PN Bl 1-D \\
PNG 358.8-00.0 & Terz N 2022 \\
PNG 359.1-01.7 & Hen 1-191, PN M 1-29, PN Sa 2-250 \\
PNG 359.2+01.2 & PN PM 1-166, 19W32 \\
PNG 359.3-00.9 & Hen 2-286, PN Bl 1-E, PN Hb 5, PN Sa 2-244 \\
\hline
\end{tabular}


Table 2. Spitzer observations of PNs in GLIMPSE II.

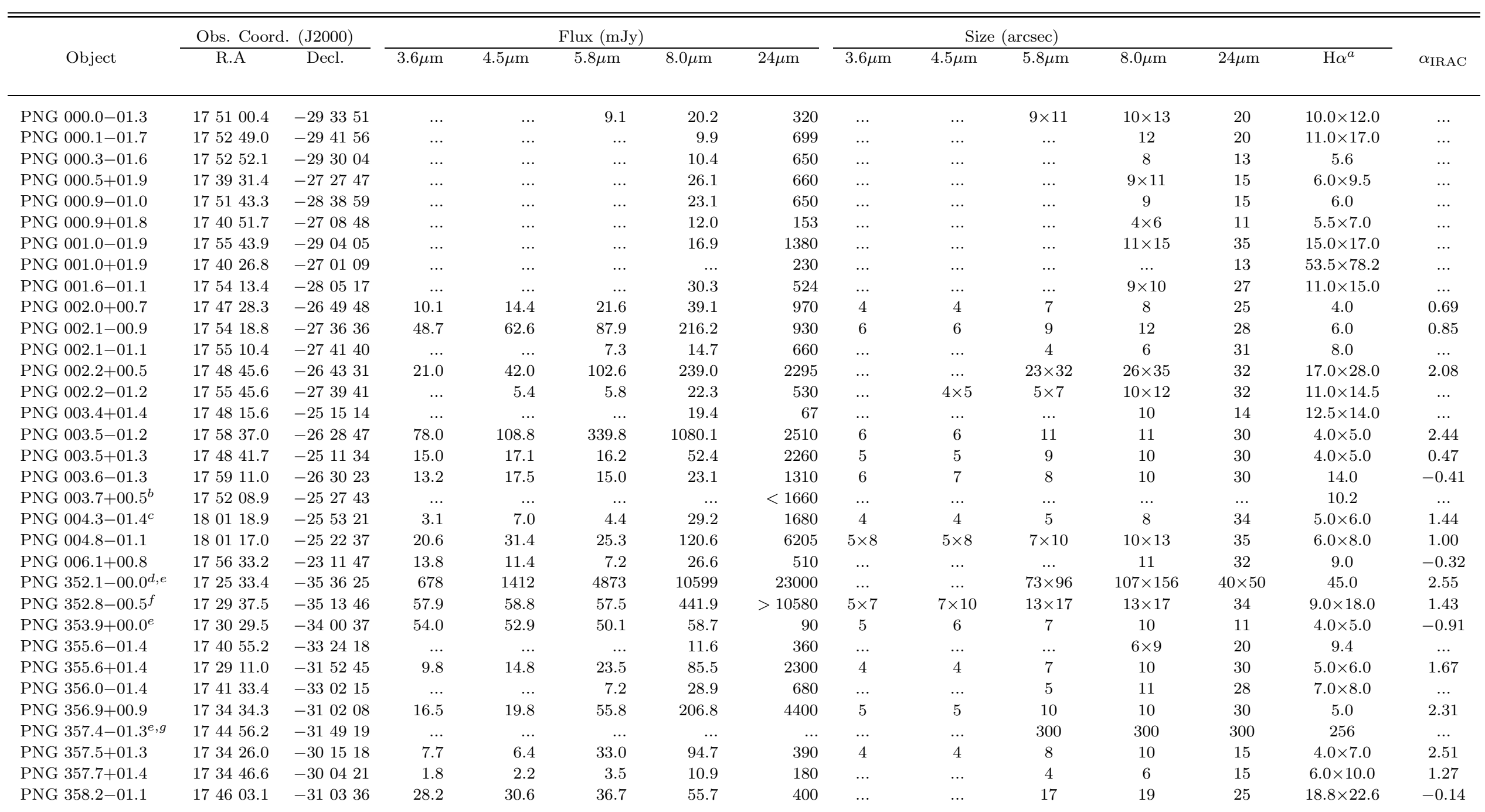


Table 2-Continued

\begin{tabular}{|c|c|c|c|c|c|c|c|c|c|c|c|c|c|c|}
\hline \multirow[b]{2}{*}{ Object } & \multicolumn{2}{|c|}{ Obs. Coord. (J2000) } & \multicolumn{5}{|c|}{ Flux (mJy) } & \multicolumn{4}{|c|}{ Size (arcsec) } & \multirow[b]{2}{*}{$24 \mu \mathrm{m}$} & \multirow[b]{2}{*}{$\mathrm{H} \alpha^{a}$} & \multirow[b]{2}{*}{$\alpha_{\text {IRAC }}$} \\
\hline & R.A & Decl. & $3.6 \mu \mathrm{m}$ & $4.5 \mu \mathrm{m}$ & $5.8 \mu \mathrm{m}$ & $8.0 \mu \mathrm{m}$ & $24 \mu \mathrm{m}$ & $3.6 \mu \mathrm{m}$ & $4.5 \mu \mathrm{m}$ & $5.8 \mu \mathrm{m}$ & $8.0 \mu \mathrm{m}$ & & & \\
\hline PNG $358.8-00.0^{e, f}$ & 174242.6 & -295135 & 2823 & 2582 & 18057 & 50662 & $>37000$ & 50 & 50 & 70 & 80 & 55 & $11.3 \times 28.3$ & 3.04 \\
\hline PNG 359.1-01.7 & 175018.0 & -303455 & 36.4 & 69.5 & 63.3 & 275.3 & 6130 & 9 & 9 & 13 & 15 & 35 & $17.0 \times 18.8$ & 1.31 \\
\hline PNG 359.2+01.2 & 173902.9 & -285637 & 442.1 & 732.0 & 1361.6 & 2119.6 & 8450 & $8 \times 16$ & $8 \times 16$ & $9 \times 17$ & $13 \times 22$ & $30 \times 42$ & $9.5 \times 26.5$ & 0.99 \\
\hline PNG $359.3-00.9^{f, h}$ & 174756.1 & -295941 & 303.1 & 312.6 & 354.8 & $>6369$ & $>18800$ & 12 & 12 & 23 & 23 & 30 & $28.3 \times 60.3$ & $\ldots$ \\
\hline
\end{tabular}

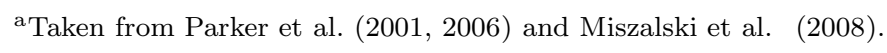

${ }^{\mathrm{b}}$ Badly blended with a bright star, which saturates all the IRAC bands.

${ }^{\mathrm{c}}$ The MIPS image is probably blended with two unresolved field stars.

d The Spitzer images reveal diffuse tail. The flux and size of the central bright region are given for the MIPS24 band.

eNon-PNs.

${ }^{\mathrm{f}}$ The MIPS images are saturated.

gIt shows large circle structure with ambiguous edge, and the given sizes are rough.

${ }^{\mathrm{h}}$ The $8.0 \mu \mathrm{m}$ image is saturated. 
Table 3. Other flux measurements

\begin{tabular}{|c|c|c|c|c|c|c|c|c|c|c|c|c|c|c|}
\hline \multirow[b]{2}{*}{ Object } & \multicolumn{3}{|c|}{ DENIS } & \multicolumn{3}{|c|}{ 2MASS } & \multicolumn{4}{|c|}{$M S X$} & \multicolumn{4}{|c|}{$I R A S$} \\
\hline & $\begin{array}{c}\mathrm{I} \\
(\mathrm{mag})\end{array}$ & $\begin{array}{c}\mathrm{J} \\
(\mathrm{mag})\end{array}$ & $\begin{array}{c}\mathrm{K} \\
(\mathrm{mag})\end{array}$ & $\begin{array}{c}\mathrm{J} \\
(\mathrm{mag})\end{array}$ & $\begin{array}{c}\mathrm{H} \\
(\mathrm{mag})\end{array}$ & $\begin{array}{c}\mathrm{K} \\
(\mathrm{mag})\end{array}$ & $\begin{array}{c}8.28 \mu \mathrm{m} \\
(\mathrm{Jy})\end{array}$ & $\begin{array}{c}12.13 \mu \mathrm{m} \\
(\mathrm{Jy})\end{array}$ & $\begin{array}{c}14.65 \mu \mathrm{m} \\
(\mathrm{Jy})\end{array}$ & $\begin{array}{c}21.3 \mu \mathrm{m} \\
(\mathrm{Jy})\end{array}$ & $\begin{array}{c}12 \mu \mathrm{m} \\
(\mathrm{Jy})\end{array}$ & $\begin{array}{c}25 \mu \mathrm{m} \\
(\mathrm{Jy})\end{array}$ & $\begin{array}{c}60 \mu \mathrm{m} \\
(\mathrm{Jy})\end{array}$ & $\begin{array}{c}100 \mu \mathrm{m} \\
(\mathrm{Jy})\end{array}$ \\
\hline PNG 002.0+00.7 & $\ldots$ & 13.902 & 11.764 & 13.858 & 12.486 & 11.82 & $\ldots$ & $\ldots$ & $\ldots$ & $\ldots$ & $\ldots$ & $\ldots$ & $\ldots$ & $\ldots$ \\
\hline PNG 002.1-00.9 & 15.616 & 14.268 & 12.009 & 13.827 & 12.702 & 11.56 & 0.159 & $\ldots$ & 1.121 & $\ldots$ & $\ldots$ & $\ldots$ & $\ldots$ & $\ldots$ \\
\hline PNG 003.5-01.2 & 16.089 & 12.894 & 10.980 & 13.020 & 12.264 & 11.03 & 0.998 & 2.653 & 2.153 & 2.266 & 1.996 & 3.438 & 9.337 & 43.230 \\
\hline PNG $003.5+01.3$ & $\ldots$ & $\ldots$ & $\ldots$ & $\ldots$ & $\ldots$ & $\ldots$ & 0.195 & $\ldots$ & 1.450 & 2.052 & $\ldots$ & $\ldots$ & $\ldots$ & $\ldots$ \\
\hline PNG 003.6-01.3 & 15.963 & 13.137 & 11.606 & 13.123 & 11.978 & 11.59 & $\ldots$ & $\ldots$ & $\ldots$ & $\ldots$ & & $\ldots$ & $\ldots$ & $\ldots$ \\
\hline PNG 004.3-01.4 & $\ldots$ & $\ldots$ & $\ldots$ & $\ldots$ & $\ldots$ & $\ldots$ & $\ldots$ & $\ldots$ & $\ldots$ & $\ldots$ & 2.272 & 1.864 & 2.691 & 3.623 \\
\hline PNG 004.8-01.1 & 17.951 & 14.203 & 12.166 & 14.333 & 12.815 & 11.71 & 0.258 & $\ldots$ & 3.162 & 5.381 & 3.329 & 8.839 & 20.550 & 106.900 \\
\hline PNG 352.8-00.5 & 18.071 & 14.654 & 12.250 & 14.995 & 13.929 & 12.24 & 0.838 & 1.774 & 4.84 & 13.878 & 1.693 & 25.430 & 137.400 & 172.900 \\
\hline PNG $353.9+00.0$ & $\ldots$ & $\ldots$ & $\ldots$ & 13.999 & 12.284 & 10.97 & $\ldots$ & $\ldots$ & $\ldots$ & $\ldots$ & $\ldots$ & $\ldots$ & $\ldots$ & $\ldots$ \\
\hline PNG 355.6+01.4 & $\ldots$ & 14.002 & 12.361 & 13.865 & 12.708 & 12.04 & 0.113 & $\ldots$ & 0.725 & 1.720 & $\ldots$ & $\ldots$ & $\ldots$ & $\ldots$ \\
\hline PNG $356.9+00.9$ & $\ldots$ & $\ldots$ & $\ldots$ & 14.495 & 13.384 & 12.34 & 0.181 & 1.946 & 0.635 & 4.195 & $\ldots$ & $\ldots$ & $\ldots$ & $\ldots$ \\
\hline PNG 358.8-00.0 & $\ldots$ & $\ldots$ & $\ldots$ & 10.375 & 9.763 & 9.285 & $\ldots$ & $\ldots$ & $\ldots$ & $\ldots$ & $\ldots$ & $\ldots$ & $\ldots$ & $\ldots$ \\
\hline PNG 359.1-01.7 & $\ldots$ & $\ldots$ & $\ldots$ & & $\ldots$ & & 0.532 & $\ldots$ & 5.100 & 4.500 & $\ldots$ & $\ldots$ & $\ldots$ & $\ldots$ \\
\hline PNG $359.2+01.2$ & $\ldots$ & $\ldots$ & $\ldots$ & 11.442 & 10.283 & 9.550 & 1.908 & 3.452 & 4.459 & 7.076 & $\ldots$ & $\ldots$ & $\ldots$ & $\ldots$ \\
\hline PNG 359.3-00.9 & 13.149 & 11.265 & 9.980 & 11.037 & 10.852 & 9.854 & 4.985 & 11.118 & 28.664 & 48.165 & 11.680 & 79.240 & 134.500 & 311.800 \\
\hline
\end{tabular}

